\title{
A Survey of Control Issues in Nanopositioning
}

\author{
Santosh Devasia, Senior Member, IEEE, Evangelos Eleftheriou, Fellow, IEEE, and \\ S. O. Reza Moheimani, Senior Member, IEEE
}

\begin{abstract}
Nanotechnology is the science of understanding matter and the control of matter at dimensions of $100 \mathrm{~nm}$ or less. Encompassing nanoscale science, engineering, and technology, nanotechnology involves imaging, measuring, modeling, and manipulation of matter at this level of precision. An important aspect of research in nanotechnology involves precision control and manipulation of devices and materials at a nanoscale, i.e., nanopositioning. Nanopositioners are precision mechatronic systems designed to move objects over a small range with a resolution down to a fraction of an atomic diameter. The desired attributes of a nanopositioner are extremely high resolution, accuracy, stability, and fast response. The key to successful nanopositioning is accurate position sensing and feedback control of the motion. This paper presents an overview of nanopositioning technologies and devices emphasizing the key role of advanced control techniques in improving precision, accuracy, and speed of operation of these systems.
\end{abstract}

Index Terms-Actuators, atomic force microscopy, nano-positioning, nanotechnology, piezoelectric devices, position sensors, servo control.

\section{INTRODUCTION}

$\mathbf{T}$ HE PAST two and a half decades have witnessed the emergence and explosive growth of nanoscience and nanotechnology [1]-[4]. The inventions of the scanning tunneling microscope (STM) [5] and the atomic force microscope (AFM) [6] have fundamentally changed research in numerous areas, including biology [7], [8], chemistry [9], materials science [10], [11], and physics [12]. Manipulation and interrogation at the nanometer scale with a scanning probe microscope (SPM) necessitate positioning systems with atomic-scale resolution [13], [14]. In SPMs, for example, nanopositioning is needed to scan the probe over a sample surface (e.g., during surface imaging) and to control the interaction between the probe and the sample surface (e.g., during surface interrogation and modification). Therefore, further advances in research in all the above mentioned SPM-based research areas and their wide applications will depend on the availability of high-precision nanopositioning stages with increasing bandwidth. Thus, it is widely recognized that one of the key requirements of nanotechnology is nanopositioning.

Manuscript received May 17, 2006; revised February 15, 2007 and June 6, 2006. Manuscript received in final form June 20, 2007. Recommended by Associate Editor K. Turner. The work of S. Devasia was supported in part by the National Science Foundation under Grant CMS 0301787.

S. Devasia is with the Mechanical Engineering Department, University of Washington, Seattle, WA 98195-2600 USA (e-mail: devasia@ u.washington.edu).

E. Eleftheriou is with the IBM Zurich Research Laboratory, CH-8803 Rüschlikon, Switzerland (e-mail: ele@ zurich.ibm.com).

S. O. R. Moheimani is with the School of Electrical Engineering and Computer Science, The University of Newcastle, Callaghan, NSW 2308, Australia (e-mail: reza.moheimani@newcastle.edu.au).

Digital Object Identifier 10.1109/TCST.2007.903345
Nanopositioning systems are also at the core of future lithography tools that might replace the ubiquitous optical lithography systems [15], [16]—it is not clear whether projection lithography, as used in the production of semiconductor integrated circuits (ICs), can still be used beyond the 35-nm technology node [17]. In general, novel nanopositioning tools are also needed for the positioning of wafers, mask alignment, and semiconductor inspection systems [17]-[19]. Furthermore, they are vital in molecular biology for imaging, alignment, and nanomanipulation as required, for example, in cell tracking and DNA analysis, nanomaterials testing [20], [21], nanoassembly [22], and the manufacturing of small objects [23]. Such nanopositioning systems are also crucial in optical alignment systems [24], [25] and even in next-generation space telescopes [26], [27]. Finally, nanometer-scale precision will soon be required for the servo system of hard-disk drives (HDD) [28], [29] and for probe-storage devices with areal densities higher than $1 \mathrm{~Tb} / \mathrm{in}^{2}$ [30], [31]. This vast range of applications with operation under such diverse conditions poses new challenges for the control of nanopositioning devices because they necessitate high resolution, high bandwidth, and robust control designs, e.g., [32]-[39].

This paper, which reviews the control-related research in nanopositioning, is organized as follows. We begin by reviewing a couple of nanopositioning applications in Section II to illustrate the control issues. This is followed by an overview of actuators and sensors used in nanopositioning in Section III before focusing on the control challenges associated with a specific actuator, i.e., piezoelectric actuators, in Sections IV and V. This focus on piezoelectric actuators is general in the sense that issues in the control of piezoelectric actuators are similar to the issues with other precision-positioning actuators. This is followed by a review of current control techniques in Section VI. Separating the control problems and control approaches into two sections (Sections V and VI, respectively) allows us to classify common control paradigms used to resolve some of the problems that are unique to nanopositioning such as creep and hysteresis. A review of emerging issues in nanopositioning is provided in Section VII.

\section{EXAMPLES OF NANOPOSITIONING APPLICATIONS}

In this section, we explain the control issues that arise in nanopositioning, using two example applications: scanning probe microscopy and dual-stage servo systems for HDDs.

\section{A. SPM}

Nanopositioning is an important aspect of a huge family of SPMs that has emerged since the invention of the STM and the AFM [33]-[39]. For example, during AFM imaging, a piezoscanner (based on piezoelectric actuators) changes the 


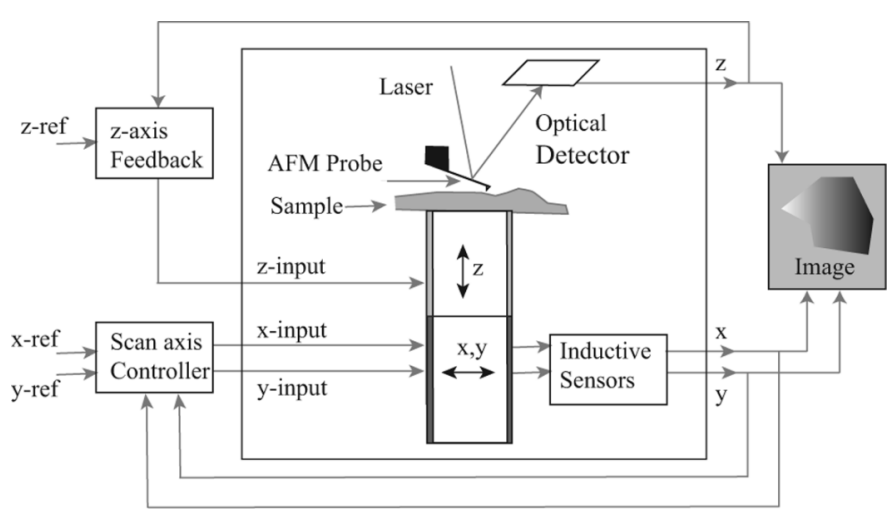

Fig. 1. AFM probe positioning. A piezoscanner is used to move the sample in the planar $x-y$ axes (parallel to the sample's substrate) and vertical $z$-axis (perpendicular to the substrate). Alternatively, the AFM probe can be moved with a piezoscanner. In some designs, separate piezoscanners are used for the vertical and planar motions.

position of the AFM probe (tip of an AFM-cantilever) relative to the specimen surface, as shown in Fig. 1.

1) Precision Positioning: Large forces between the AFM probe and the sample surface can damage the sample or the probe. To prevent this, the force between the AFM probe and the sample surface is controlled using a feedback loop: First, the applied probe-sample force is estimated by measuring the deflection of the AFM cantilever (which is proportional to the probesample force) with optical detectors, e.g., see [40]. Second, the measured cantilever deflection is used in a feedback loop to adjust the vertical position $(z)$ of the AFM probe (on the order of the instrument's resolution) such that the cantilever deflection is maintained at a desired value. Thus, by maintaining a constant (small) cantilever deflection, a constant (small) probe-sample force is maintained during imaging. In this sense, precision positioning of the AFM probe is needed to maintain the tip-sample force at the desired level during AFM imaging.

2) Obtaining an AFM Image: Changing the vertical position of the AFM probe to maintain a constant tip-sample interaction (force) is known as constant force and usually yields a fairly faithful topographical image. An image of the sample's topography is obtained by first raster-scanning the probe over the sample and then plotting the $z$-displacement of the AFM probe (estimated using the voltage $V_{z}$ applied to position the AFM probe in the $z$-direction) against the AFM probe's $x-y$ position relative to the substrate on which the sample is placed (see, e.g., [41]). The resulting image resembles an image on a television screen in that both consist of many rows or lines of information placed one above the other.

3) Precision Positioning in Other Operating Modes: Other modes of AFM operation include the tapping mode, which maintains a fixed amplitude of oscillation in the AFM cantilever rather than maintaining a constant force. Moreover, many new variants of SPM exist that allow the measurement of local chemical, mechanical (frictional), electric, and magnetic properties of a surface (e.g., [14], [42], [43]). In all of these variants, nanopositioning is important to precisely position the SPM probe in close proximity to the sample surface.

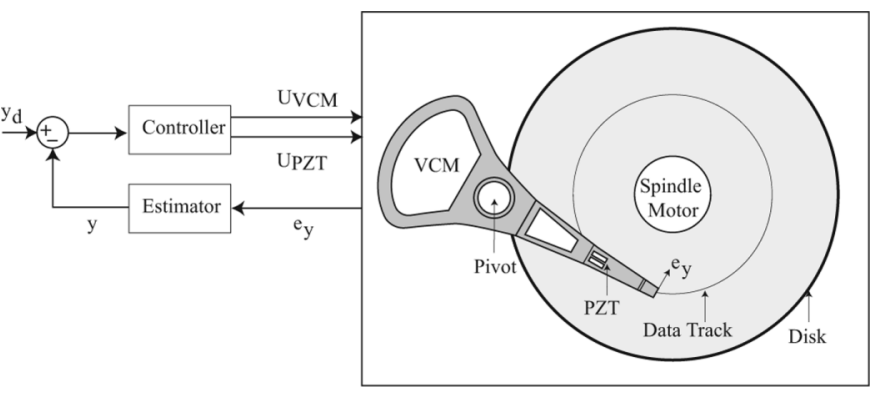

Fig. 2. Block diagram of servo system for an example dual-stage HDD. The error in head position $e_{y}$ over a target data track is used to estimate the position $y$ of the head. The input $U_{\mathrm{VCM}}$ to a VCM and $U_{\mathrm{PZT}}$ to a piezoelectric (PZT) actuator are used to position the head at a desired position $y_{d}$. During track follow, the position $y$ is maintained over a single data track; during track seek, the goal is to change the position from one data track to another.

4) Increasing the Positioning Bandwidth: SPMs allow scientists to image topography or measure the local property of the specimen under investigation at high resolution, albeit only at relatively low speed. The low speed is primarily attributed to the limited bandwidth of the PI-controlled SPM, which is determined by the dynamics of the piezoelectric scanner. High-bandwidth operation is imperative when imaging the time-varying nature of the specimen under investigation, e.g., for imaging the movements of a living cell or the formation of surface features during materials processing. Lack of high-speed imaging implies that the resulting AFM images have substantial distortions because the sample has changed considerably while the image was being collected. Therefore, a current challenge in SPM is to achieve the nanopositioning of the SPM probe with high bandwidth.

\section{B. Dual-Stage Servo Systems for HDDs}

Nanopositioning of the READ-WRITE head over data tracks is becoming an important issue in HDDs as the data tracks become smaller because of the ever increasing areal density of HDDs. Today, a typical track width is on the order of $200 \mathrm{~nm}$, a figure that is expected to decrease annually by a factor of approximately $30 \%$. A simple rule of thumb for servo design in HDDs is that three times the statistical standard deviation of the position error between the head and the center of the data track should be less than one-tenth of the track width. To achieve these future ultra-high areal densities, nanometer-level precision of the servo system is required.

1) Precision Positioning: The positioning problem is to place the READ-WRITE head over a desired spot on the hard disk for recording and retrieving data to and from an HDD (see Fig. 2). Currently, this positioning is achieved by two actuators: a spindle motor that rotates the disk and a voice-coil motor (VCM) that sweeps the head radially over the disk surface. The head position relative to the target track center is monitored and adjusted periodically by reading servo information embedded in the data tracks, using a closed-loop control system that drives the VCM actuator. It is noted that a precision positioning servo is important to READ and WRITE data at the intended location on the disk; however, nonlinear friction of the pivot bearing limits the achievable servo precision. Moreover, the bandwidth 


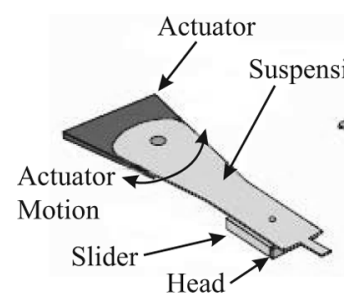

(1)

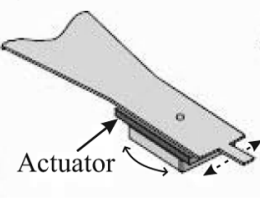

(2)

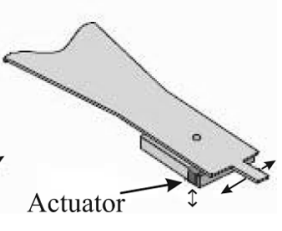

(3)
Fig. 3. Dual-stage servo configurations. Reprinted with permission from [51], (C) 2003 by ASME. (1) Actuated suspension. (2) Actuated slider. (3) Actuated head.

of a traditional single-stage servo system is limited by the multiple mechanical resonances of the actuator as well as by input-magnitude constraints [44], [45].

2) Dual-Stage Positioning Systems: Dual-stage actuation has been proposed as a solution that would increase both servo bandwidth and precision [46]-[50]. In such a system, the VCM acts as a first-stage coarse positioner and the microactuator, which rides on the moving arm of the VCM, is used as a secondary fine actuator. Note that the second stage is located closer to the READ-WRITE head (on the moving arm) and has relatively less mass to move; therefore, it tends to have a higher bandwidth than the VCM. In addition, the second actuator tends to have a significantly higher positioning precision than the VCM. Thus, the integrated dual-stage system has the large range of the VCM as well as the high precision and large bandwidth of the second stage.

3) Types of Secondary Actuation: Currently, three main types of secondary actuation systems have been proposed: actuated suspension, the actuated slider, and the actuated head (see Fig. 3). In all these configurations, the actuation forces are generated by piezoelectric, electrostatic, or electromagnetic microactuators [29], [51]. For example, in the actuated-suspension approach, the microactuator is positioned around the suspension base and actuates the entire suspension (and slider) for head positioning [28], [52], [53]. The advantage of an actuated suspension is that standard fabrication processes can be used for attaching the piezoelectric secondary actuator to the base of the suspension; however, a disadvantage is that the increase in servo bandwidth is limited by the resonant modes of the suspension. By moving the microactuator closer to the READ/WRITE element (thereby reducing the mass that needs to be moved) the actuated-slider approach, with the microactuator between the slider and the suspension, results in increased bandwidth [54], [55]. The microactuator is closest to the READ/WRITE element in actuated-head designs, where the microactuator is placed between the slider body and the READ/WRITE element. One of the most critical challenges of this approach is that the actuator fabrication process must be compatible with those of the slider and the head [56].

4) Positioning Control Issues: To introduce this technology into commercial products, several aspects still require further research, namely, system integration, reliability, and performance enhancement. Two of the largest commercialization obstacles to be overcome at this time are the cost and reliability of actuated-slider dual-stage servo systems. Other aspects that are the focus of further research are the dynamic behavior and reliability of the microactuator subjected to disturbances due to airflow, the interaction between head and disk, and the presence of contaminants or particles. Future dual-stage servo systems may well employ an actuated head approach to achieve nanometer servo precision at high bandwidth. Here, research has just begun and it focuses on the key problem of how to combine the fabrication processes of microactuators and READ/WRITE heads. Also, being investigated is the use of micro-electromechanical systems (MEMS) technology to incorporate additional sensors, such as accelerometers and strain gage vibration sensors, to suppress airflow-induced track miss-registration and external disturbances due to mechanical vibrations. Moreover, such a multisensing and multiactuation servo system also requires new, robust, adaptive multiinput-multioutput (MIMO) control architectures and algorithms [29]. Positioning control problems for the dual-stage system include track seek, where the objective is to transition from one track to another, and track follow, i.e., maintaining the position over a specified track [57]-[59].

\section{ACTUATORS AND SENSORS IN NANOPOSITIONING}

An important issue in the design of controllers for nanopositioning is the availability and choice of actuators and sensors. Therefore, this section reviews some of the actuators and sensors used in nanopositioning.

\section{A. Actuators for Nanopositioning}

The main design parameters that characterize the performance of an actuator are displacement range, force, bandwidth (frequency range), size, weight, and power consumption. Most actuators usually perform well in some of these categories, but typically poorly in others. Hence, Section III-A reviews the advantages and disadvantages of actuation approaches used for nanopositioning.

1) Piezoelectric Actuators: Piezoelectric actuators are ubiquitous in nanopositioning applications such as SPMs and micromotors. They have excellent operating bandwidth and can generate large mechanical forces in a compact design and for small amounts of power, but they have a relatively small displacement range. Bulk ceramic piezoelectric devices based on lead zirconate titanate (PZT) are already well established, but recently also thin-film-based devices have started to appear in many applications [60], [61]. Issues in the modeling and control of piezoelectric actuators are discussed in Sections IV and V.

2) Magnetostrictive Actuators: In magnetostrictive or piezomagnetic material, magnetization in an external field which induces a dimensional change of the material [62]. Rods of magnetostrictive materials have found use in the industry in the form of actuators because of their ability to convert electrical energy into mechanical energy. Typically, current passing through a coil located around a magnetostrictive rod produces the necessary magnetic field for actuation. Precision positioning can be achieved by precise control of the current. This actuation mechanism is important when large forces must be obtained over small distances. Terfenol-D is a well-known material with a high magnetostriction coefficient that was developed in the 1960s. Sputter-deposited magnetostrictive films present an interesting opportunity for actuation in micromachines that re- 

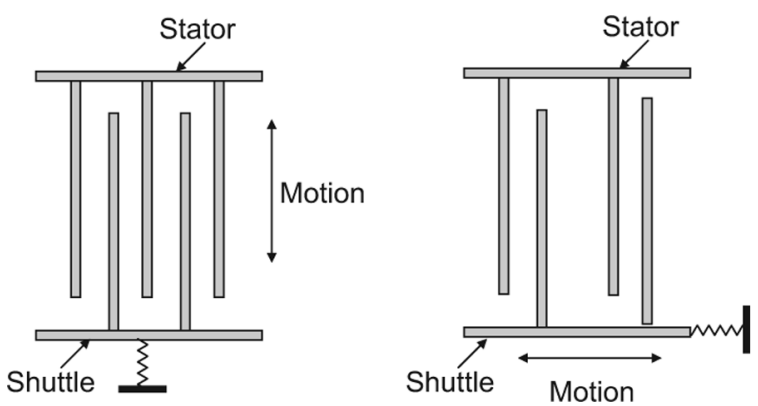

Fig. 4. Electrostatic microactuators: (left) interdigitated comb actuator and (right) parallel-plates actuator.

quire contactless high-frequency operation. The power requirements for magnetostriction are greater than for piezoelectric materials, but the actuation offers a larger displacement and the ratio of mass per unit stress is greater than with a PZT actuator. As in piezoelectric actuators, control issues in magnetostrictive actuators include addressing hysteresis effects [63], [64]. Another approach with magnetic fields is to levitate an object; nanometer-scale precision is also achievable with such magnetic levitation systems [65].

3) MEMS-Based Electrostatic Actuator: Electrostatic microactuators are well established in the MEMS field because of their ease of fabrication as only the structural material needs to be conductive. The electrostatic actuators consist of two interdigitated comb structures, one being fixed (the stator), the other movable (the shuttle). An electrical potential applied between the two combs generates a force either in the direction that increases the overlap of the fingers or in the direction that increases the distance between the electrodes, as illustrated in Fig. 4. The former is called interdigitated comb actuator, the latter parallel-plates actuator.

Interdigitated comb and parallel-plates actuators are, in principle, easy to fabricate- a single mask level and one deep-reactive-ion-etch (DRIE) step suffice to delineate the combs, the table, the springs, and the frame. For closed-loop position control, the position of the shuttle is determined by measuring the capacitance across a pair of combs. Electrostatic microactuators have been intensively explored as secondary actuators in dualstage servo systems of HDDs, particularly in the actuated-slider and actuated-head configurations (see [29] and the references therein). They have also been proposed for the medium-actuator design of probe-storage devices [66]. Drawbacks of interdigitated comb drives, however, are the relatively weak force they generate and the high voltage they need. For example, for a 50- $\mu \mathrm{m}$ motion with 200 fingers, each being $500 \mu \mathrm{m}$ high and spaced $16 \mu \mathrm{m}$ apart, as much as $120 \mathrm{~V}$ may have to be applied [66].

4) MEMS-Based Electrostatic Surface Actuator: This type of actuator (see Fig. 5), which employs a principle similar to a stepper motor [67], also consists of two periodic electrode structures: one fixed (to the stator) and the other movable (on the translator). In contrast to an interdigitated comb drive, however, the electrodes are planar and face each other. To move the translator to stable positions, in which the periodic structures are aligned, in-plane forces are generated between them. These forces are determined by the spatial phase relationship between

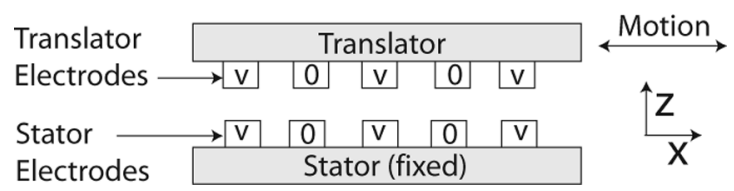

Fig. 5. Electrostatic surface actuator. An example voltage pattern is shown on the electrodes; the voltage pattern is varied to move the translator.

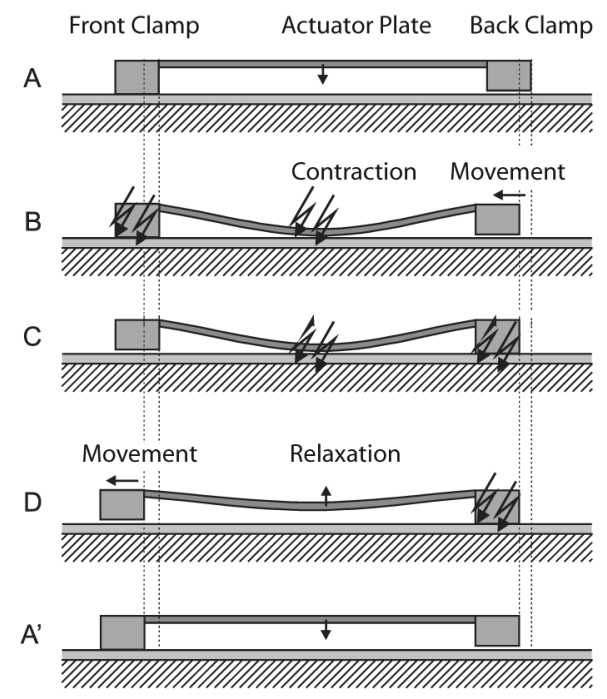

Fig. 6. Motion cycle of 1-D shuffle actuator [70]. Adapted with permission from [71].

the two sets of periodic electrodes and the voltages applied to them. For continuous motion, each surface has multiple interleaved periodic electrodes that can be successively activated to create a voltage wave to move the translator in the desired direction. However, this type of actuator also exerts large $z$-axis forces, which must be taken into account in the design of flexure springs used to hold the translator above the stator.

Here, as in the case of other stepping-type actuators, the position of the translator is known to within a single step without an independent position sensor unless the actuation force is overcome by an external force. For an actuator of this type that has seven addressable periodic electrodes, a position resolution as small as $5 \mathrm{~nm}$ has been achieved [68]. The intrinsic electrostatic centering force at each step position acts as a restoring force. In operation, the overall in-plane stiffness of the translator can be much greater than the stiffness of the flexure springs needed to hold the translator in place. Accordingly, relatively soft flexure springs can be used for better power efficiency if a strong holding force is maintained to counter external disturbances. In other actuation systems, this is accomplished via closed-loop servo, which, however, requires an independent position sensor and control system [69].

5) MEMS-Based Electrostatic Shuffle Actuator: The shuffle actuator (also known as inchworm actuator) is an electrostatic design based on a clamp and slip motion. This design consists of two feet, independently clamped to the substrate via electrostatic force, and a membrane that can be reversibly collapsed by an electrostatic force [70]. Its motion cycle is described in Fig. 6. As the moving element is always clamped by at least one foot, it exhibits high in-plane and out-of-plane rigidity for immunity 

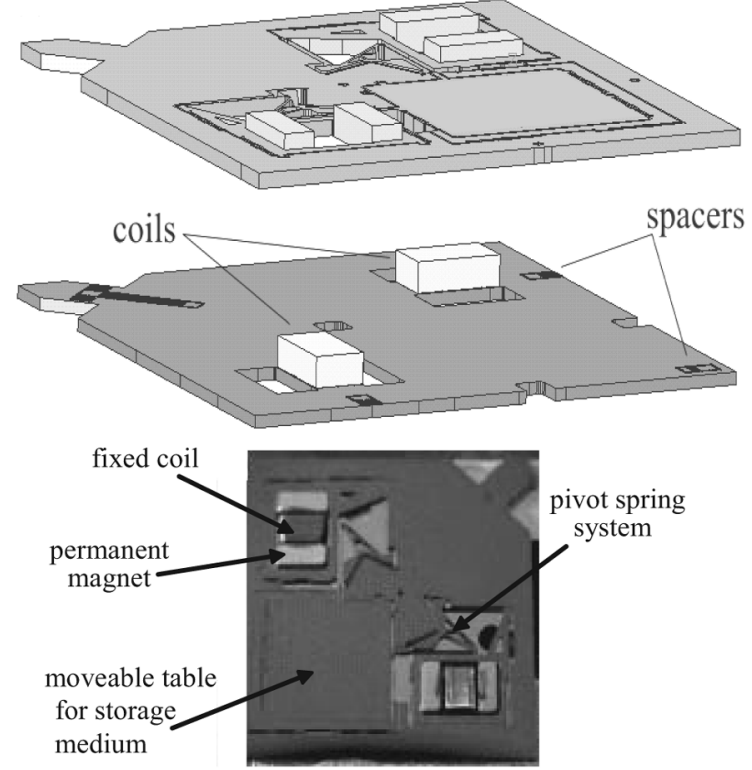

Fig. 7. (top) Exploded view of the nanopositioner with base plate assembly. The nanopositioner with the magnets is visible above the base plate and coils. (bottom) Photograph of an assembled prototype nanopositioner. Reprinted with permission from [74].

to external disturbances without requiring a stiff flexure and the ensuing power consumption penalty. So far, only 1-D devices have been built with this concept [69]; however 2-D versions are conceivable. One of the potential problems of such an approach could be the wear due to the shuffling motion.

6) MEMS-Based Electromagnetic Actuator: Here, actuation generates a force via the flow of current through a wire coil in the presence of a magnetic field. This concept is widely used because of its high force and efficiency in conventional macroscopic actuators. Conventional fabrication methods using wirewound coils can be used to build efficient miniature electromagnetic motors and actuators, even for devices as small as $1 \mathrm{~mm}$ [69]. Although from a fabrication point of view, planar integrated coils and magnets created by depositing magnetic films would appear to be attractive in MEMS scanners, designing and fabricating them with a sufficient number of coil turns and a sufficient mass of magnetic material to generate large forces efficiently is difficult. Therefore, hybrid solutions, in which MEMS techniques are used to fabricate the passive mechanical components such as the frame, flexures, and table, and conventional discrete magnets and wire-wound coils serve as the active elements, are often used instead [72]-[74].

An advanced hybrid design of an electromagnetic-actuator-based nanopositioner is shown in Fig. 7, in which actuation is achieved by using two voice-coil-type actuators [74]. This method of actuation provides a large travel range at low operating voltages, compared with electrostatic or piezoelectric actuation. The actuators are assembled in the rectangular frames shown in the upper left and lower right of Fig. 7(bottom). Each actuator consists of a pair of rare earth $(\mathrm{NdFeB})$ magnets mounted in the actuator frames using epoxy glue. The two magnets within an actuator are mounted with their magnetization aligned with the central axis of the coil, but pointing in opposite directions. Thus, when a current is applied to the coil, one magnet is attracted to the coil and the other is repelled by it, resulting in forces on the shuttle in the same direction. The net force results in a displacement of the shuttle that is related to the stiffness of the positioner. Reversing the direction of the current generates force and displacement in the opposite direction. The magnitude of the force generated on a single magnet is a nonlinear function of the spacing between the magnet and coil. However, by using two magnets in this push-pull configuration, a net force is generated that, for small displacements, is approximately constant over the different displacements of the shuttle. Combined with the linear spring system of the positioner, this results in a displacement of the actuator that is a linear function of the applied current.

The mechanical structure is fabricated from a $400-\mu \mathrm{m}$-thick single-crystal silicon ( $\mathrm{Si}$ ) wafer using a DRIE process. The fixed coils are located between opposing magnets attached to moving shuttles. Note that in this design, pivoting reversing levers between the actuator and table are used for both axes of motion: in this way, a motion of the actuator results in a motion of the scan table in the opposite direction. This rotational coupling allows the masses on each side of the reversing lever to be balanced. In such a "mass-balanced" design, the external acceleration forces on the table and shuttle cancel one another, rendering the system robust against external shock and vibration [74], [75]. Although mass-balancing is common in rotary actuators, it is less often applied to linear actuators because of the complexity of the mechanism needed. In a MEMS scanner, this complex mechanism can be fabricated with high precision and at low cost. The planar electromagnetic nanopositioner, shown in Fig. 7 , has $x, y$-motion capabilities of $\pm 60 \mu \mathrm{m}$ with an average power consumption of 10 to $20 \mathrm{~mW}$ per axis. The nanopositioner achieves linear displacement as a function of the driving current and exhibits a crosstalk between axes of $1 \%-3 \%$. This design is suitable for parallel probe-based mobile storage devices or as a generic nanopositioning system for other nanotechnology applications.

7) MEMS-Based Thermal Actuator: The driving mechanism in thermal actuation is either the bimorph effect or the use of hot-cold arms [76]-[78]. In the latter approach, microstructures with single-layer material can produce in-plane motion because of the difference in thermal expansion of a narrow hot arm and a wide cold arm. MEMS-based thermal actuators for nanopositioning applications have recently attracted renewed research interest-not only because they generate large deflection and force but also because their fabrication process is compatible with the standard CMOS process. For example, in [78] a MEMS hot-cold-arm thermal actuator design is described with bidirectional vertical motion. This design is a U-shaped structure that provides a larger deflection and better electrical efficiency than the traditional vertical thermal actuator does, owing to the use of an active return current path.

Irrespective of the physical effect used for actuation, thermal actuators are in principle sensitive to the ambient operating temperature and thus their use in nanopositioning applications may be limited. Very recently, a multimorph thermal actuator fabricated in a standard CMOS process was presented in [79]. In this design, a temperature-insensitive capacitive positioning mechanism has been incorporated that allows position detection with 
respect to large displacements. The thermal actuator achieved a $24-\mu \mathrm{m}$ displacement with a power consumption of $17 \mathrm{~mW}$. For a closed-loop control bandwidth of $1 \mathrm{kHz}$, the sensor noise would limit the minimum detectable signal to $5 \mathrm{~nm}$. So far for this concept, only a 1-D device has been built; however, 2-D versions are conceivable.

\section{B. Sensors for Nanopositioning}

The speed and absolute positioning accuracy of various nanopositioning systems crucially depend on the position-sensing mechanism. On the other hand, generation of nanometer-scale position information is in itself challenging, particularly in the wide bandwidth and large dynamic range required to achieve a reasonable throughput in many nanotechnology applications, including lithography and data storage. Sensors based on a variety of techniques [80], such as inductive [81], piezoresistive [82], capacitive [29], and optical measurements [40], [83], [84] are suitable for nanopositioning applications. In this review, we focus on two position-sensing techniques that are easily integrated with arrays of MEMS-based nanopositioning actuators, namely, capacitive position sensing and thermal position sensing. Capacitive sensing is one of the most popular position-sensing techniques in conjunction with microactuators that achieve nanometer resolution. Very recently, silicon displacement sensors based on the conduction of heat between two surfaces through the ambient air have also demonstrated displacement resolutions of less than $1 \mathrm{~nm}$.

1) Capacitive Position Sensors: Position transducers based on capacitive sensing are very popular in the industry because they provide a relatively simple technique to implement a noncontact measurement. The principle of capacitive position sensing is based on the movement of the shuttle electrode that causes a capacitance change between the fixed (stator) and the movable electrode (shuttle). By measuring the change in capacitance the shuttle location relative to the stator can be determined. After conversion from capacitance to voltage by means of an electronic circuitry, the capacitance change due to shuttle movement provides an output voltage that is a function of the shuttle's displacement. It can be shown that, for the comb-drive configuration, the output voltage and the shuttle displacement are linearly related. On the other hand, in the case of the capacitive sensing approach that uses the parallel-plate drive arrangement, the output voltage and the shuttle displacement are nonlinearly related. This nonlinear behavior can be linearized, to a certain extent, by using a differential parallel-plate configuration [29]. For electrostatic microactuators that use capacitive sensing for closed- or open-loop position control, the capacitance variation is on the order of $100 \mathrm{fF} / \mu \mathrm{m}$ [29]. Clearly, to achieve nanometer position resolution, the circuitry must be able to detect capacitance variations on the order of $0.1 \mathrm{fF}$ in the presence of parasitic capacitances. It is well known that for capacitive position sensors the sensing circuit design is the most critical component.

2) Thermal Position Sensors: The basic design of the thermal position sensor uses a short, elongated U-shaped, free-standing cantilever as shown in Fig. 8(a) [85]. The devices (a)

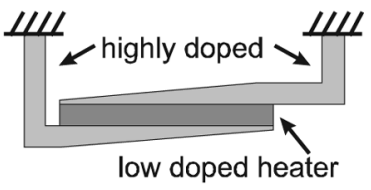

(b)

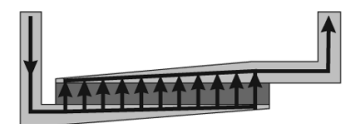

Fig. 8. (a) Schematic of thermal position sensor. (b) Current flow through the resistive heater results in a rise in temperature of the device. The black arrows indicate the current path through device. Reprinted with permission from [85], (C) 2005 IOP Publishing Ltd.

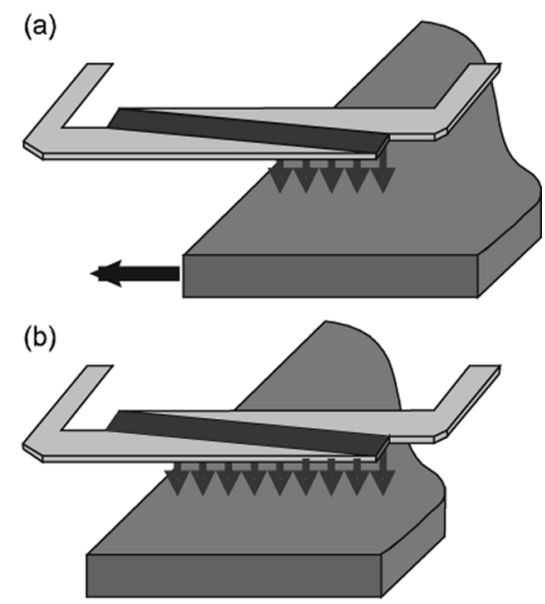

Fig. 9. (a) Heat conduction through the air into the underlying surface. (b) Displacement of underlying surface towards the left results in an increase in the cooling efficiency, implying a decrease in the temperature of the heater, which in turn gives rise to a decrease in its electrical resistance. Reprinted with permission from [85], (C) 2005 IOP Publishing Ltd.

are made from single-crystal Si using standard bulk micromachining technology. The sensing element is a resistive heater made from moderately doped $\mathrm{Si}$ and supported by legs made from highly doped Si that act as electrical leads. Application of a voltage across the legs of the device results in a current flowing through the heater and a subsequent increase in the device temperature, as illustrated in Fig. 8(b). To use this device as a displacement sensor, it should be positioned directly above an edge or step on the object of interest, with the long axis of the heater aligned parallel to the axis of motion. In addition, the edge should be perpendicular to the direction of motion to be measured and the surface of the sensor should be parallel to that of the object of interest, as illustrated in Fig. 9. To sense a displacement of the object, the temperature dependence of the Si resistance is exploited. A fraction of the heat generated by the resistive heater is conducted through the ambient air into the scan table, which acts as a heat sink. Displacement of the scan table gives rise to a change in the efficiency of this cooling mechanism, resulting in a change in the temperature of the heater and thus a change in its electrical resistance. The sensors are driven by a constant voltage, and changes in the resulting current using a current-to-voltage $(I / V)$ amplifier are measured. To minimize drift effects, the sensors are operated in pairs using a differential configuration. Although these devices 
are quite simple, the linearity, noise limit, and drift performance that can be achieved are surprisingly good. In particular, a displacement resolution of less than $1 \mathrm{~nm}$ and a dynamic range of more than $100 \mu \mathrm{m}$ were achieved in a $10-\mathrm{kHz}$ bandwidth. Power consumption of these devices is on the order of $10 \mathrm{~mW}$ per sensor, and the measured time response is described by a simple exponential with a time constant of approximately $100 \mu \mathrm{s}[85]$.

\section{Piezoelectric ACtUATORS FOR NANOPOSITIONING}

This section discusses the use of piezoelectric materials for the design of nanopositioning actuators. The focus is on piezoelectric actuators because they are omnipresent in nanopositioning applications, ranging from relatively small-range nanopositioning stages of SPM to larger-range motors, e.g., [86] and [87]. Issues in the control of piezoelectric actuators, such as bandwidth and resolution improvements by overcoming actuator dynamics that includes creep, hysteresis nonlinearities, and vibrations, are similar to the issues in other precision-positioning actuators, such as magnetostrictive actuators.

\section{A. Piezoelectric Actuators}

The piezoelectric effect was first discovered by the Curie brothers in 1880 [60], [61]. Specifically, they found that squeezing certain materials (piezein is the Greek word for squeeze) results in an electric charge; this effect enables the use of piezoelectric materials in strain sensors. However, the use of piezoelectric materials as actuators exploits the converse effect, i.e., that the application of an electric voltage results in a mechanical strain. This converse effect is credited to Lippmann's theoretical predictions, which were also experimentally verified by the Curie brothers. Piezoelectric actuators made of ceramic PZT have been used for a long time, but recently thin-film actuators have started to appear in many applications. Historical perspectives of the piezoelectric effect as well as recent advances in ceramic and thin-film actuators are provided in [60], [61], and [88].

1) Fabrication of Piezoelectric Actuator: A piezoelectric ceramic is a mass of perovskite crystals, in which each crystal is composed of a small, tetravalent metal ion placed inside a lattice of larger divalent metal ions and $\mathrm{O}_{2}$ [89]. To prepare a piezoelectric ceramic, fine powders of the component metal oxides are mixed in specific proportions. This mixture is then heated to form a uniform powder, which in turn is mixed with an organic binder and formed into specific shapes, e.g., discs, rods, plates, tubes, etc. These elements are then heated for a specific time and under a predetermined temperature. In this process, the powder particles sinter and the material forms a dense crystalline structure. The elements are then cooled and, if needed, trimmed into specific shapes. Finally, silver or copper electrodes are applied to the appropriate surfaces of the structure.

2) Poling the Piezoelectric Actuator: Above a critical temperature, known as the "Curie temperature," each perovskite crystal in the heated ceramic element exhibits a simple cubic symmetry with no dipole moment. However, at temperatures below the Curie temperature, each crystal possesses a dipole moment. Adjoining dipoles form regions of local alignment called "domains." This alignment gives a net dipole moment

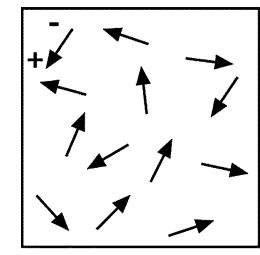

(a)

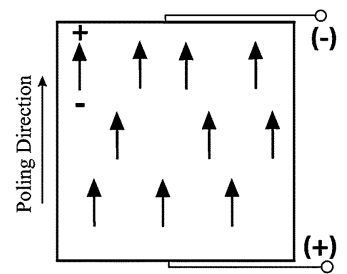

(b)

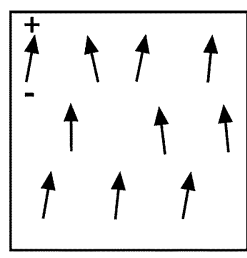

(c)
Fig. 10. Poling process: (a) Prior to polarization polar domains are oriented randomly. (b) A very large dc electric field is used for polarization. (c) After the dc field is removed, the remnant polarization remains.

to the domain, and thus a net polarization. As demonstrated in Fig. 10(a), the direction of polarization among neighboring domains is random. Subsequently, the ceramic element has no overall polarization.

The domains in a ceramic element are aligned by exposing the element to a strong dc electric field, usually at a temperature slightly below the Curie temperature [see Fig. 10(b)]. This is referred to as the "poling process." After the poling treatment, those domains that are almost aligned with the electric field expand at the expense of domains that are not aligned with the field and the element expands in the direction of the field. When the electric field is removed, most of the dipoles are locked into a configuration of near alignment [see Fig. 10(c)]. The element now has a permanent polarization, the remnant polarization, and is permanently elongated. The increase in the length of the element, however, is very small, usually in the micrometer range.

3) Modeling the Piezoelectric Effect: If a poled piezoelectric ceramic element is mechanically strained, a resulting electric charge is produced in the material. This is often referred to as the "direct piezoelectric effect" and is illustrated in Fig. 11(a). Conversely, application of an electric field to the element results in the material being mechanically strained. This property is known as "converse piezoelectric effect," and is shown in Fig. 11(b).

The electromechanical equations for linear piezoelectric material can be written as (see [90] and [91, Ch. 5])

$$
\begin{aligned}
S_{i} & =s_{i j}^{E} T_{j}+d_{m i} E_{m} \\
D_{m} & =D_{m i} T_{i}+\epsilon_{m k}^{T} E_{k}
\end{aligned}
$$

where the indexes $i, j=1,2, \ldots, 6$ and $m, k=1,2,3$ refer to different directions within the Cartesian coordinate system, as shown in Fig. 12. The first index refers to the stimuli direction (e.g., electric field), the second shows the direction of reaction (e.g., strain). $S, T, D$, and $E$ correspond to the mechanical strain, stress, electrical displacement, and electric field, respectively. The material properties $s^{E}, d$, and $\epsilon^{T}$ represent the elastic, piezoelectric strain, and dielectric constants. The second equation describes the direct and the first the converse piezoelectric effect. By convention, the " 3 " axis in Fig. 12 is aligned with the poling direction.

When the applied electric field $(E)$ has the same polarity as the poling direction, the resulting strain will be positive, which corresponds to an elongation, whereas if they are opposite the resulting stain will be negative, which corresponds to a contraction. 


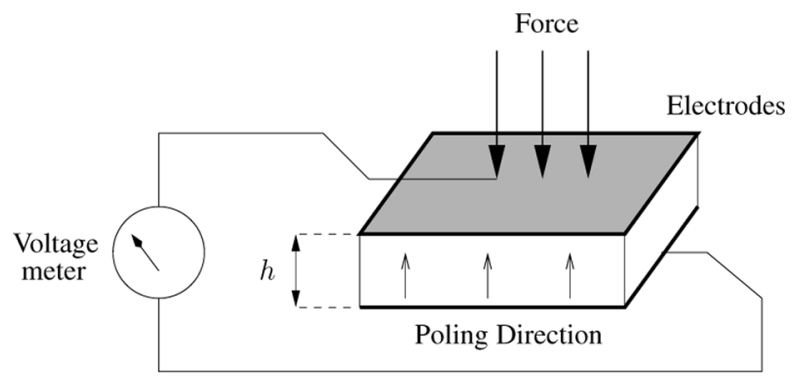

(a)

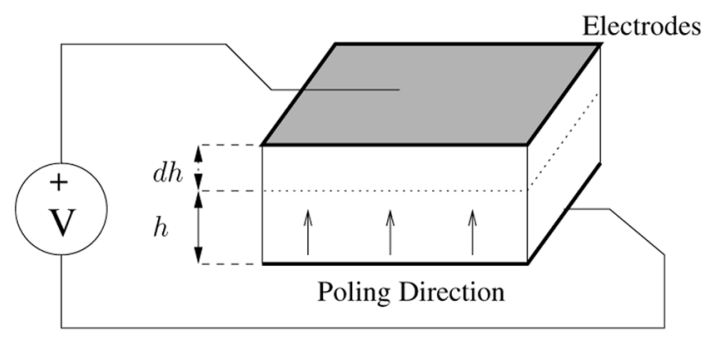

(b)

Fig. 11. Direct and converse piezoelectric effect. (a) Direct effect. (b) Converse effect.

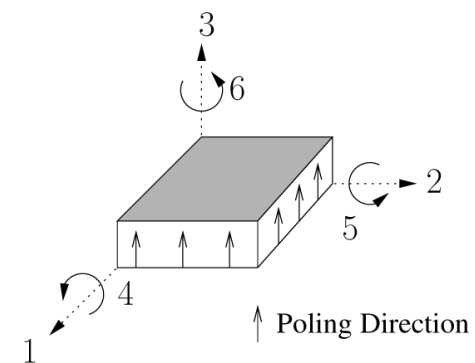

Fig. 12. Right-hand Cartesian coordinate.

For a piezoelectric element, all parameters in (1) and (2) are tensors. However, for objects of certain regular shapes (plates, shells, etc.) these equations can be substantially simplified thanks to the geometrical symmetries of these objects and specific properties of the piezoelectric materials used in their construction [89], [92].

\section{B. Piezoelectric Tube Scanners}

The use of piezoelectric tube scanners in SPM was first reported in [93]. Compared with traditional tripod positioners, these actuators were found to provide a higher positioning accuracy and larger bandwidth. In addition, they proved to be easier to manufacture and to integrate into a microscope. Piezoelectric tube scanners are now used extensively in SPM and many other applications that involve nanopositioning [36], [94]-[97].

1) Schematic of Positioning Operation: As illustrated in Fig. 13, a piezoelectric tube scanner consists of a tube of radially poled piezoelectric material, four external electrodes, and a grounded internal electrode. Opposite electrodes are segmented in four equal sectors of $90^{\circ}$ and driven by signals of identical magnitude, but opposite signs. Through bending, this results in a 2-D movement in the $x-y$ plane on, approximately, a sphere. In this configuration, the inner electrode is driven by the $z$-signal. Another configuration includes a circumferential outer electrode for independent vertical extension or contraction as illustrated in Fig. 1. A drawback of using outer electrodes for $x-y-z$ movement is that a large scan size reduces the available range for $z$-control.

2) Positioning Range: Precise calculation of the scanning range of a piezoelectric tube can be quite difficult [98]-[100]. The bending of the tube is a function of the electric field and the
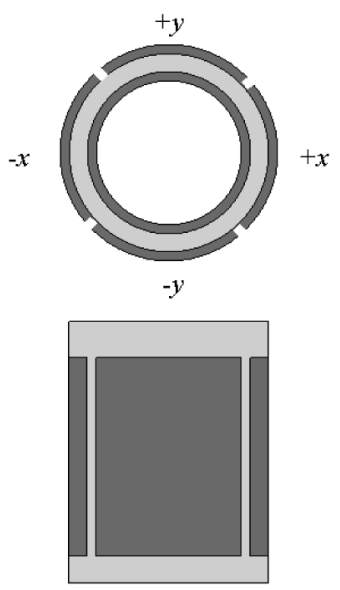

Fig. 13. Top and side view of a piezoelectric tube scanner with four external electrodes and a continuous inner electrode. The bottom end of the tube is fixed and the top end is used for positioning purposes. Equal, but opposite, voltages are applied to opposite external electrodes to achieve $x-y$ displacement, i.e., bending of tube. By applying a voltage to the inner electrode $z$-positioning (elongation of the tube) can be achieved.

induced nonuniform strain. Assuming small deflections, however, would result in the following approximate expression for the tip translation along the $x$ - or $y$-direction [100]

$$
\Delta_{i}=\frac{\sqrt{2} d_{31} L^{2}}{\pi D h} v_{i} \quad i=x, y,
$$

where $\Delta_{i}$ is the deflection in the $x$ - or $y$-direction, $d_{31}$ is the piezoelectric strain constant, $L$ is the tube length, $D$ is the outside diameter of the tube, $h$ is the tube thickness, and $v_{i}$ is an $x$ - or $y$-axis electrode voltage. The tip deflection can be doubled by applying an equal, but opposite, voltage to the electrodes in the same axis. Vertical translation due to a voltage $v_{z}$ applied to the inner electrode is approximately given by

$$
\Delta_{z}=\frac{2 d_{31} L}{h} v_{z}
$$

\section{Modeling AND CONTROL Issues}

Due to the key role played by piezoelectric actuators (e.g., piezoelectric tubes in SPMs) and the increasing interest in using model-based control design techniques to improve the precision and scanning speed of these devices [33]-[37], [101], [102] interest in understanding the dynamics of these actuators has increased. A number of studies have been performed in this 
area, e.g., [103]-[108] and the references therein. The challenges of the modeling and control of piezoelectric actuators are discussed in this section.

\section{A. Creep}

Creep is an undesirable property common with piezoelectric actuators. It can result in significant loss in precision when positioning is required over extended periods of time [109]. In particular, during slow operation of SPMs, creep can result in significant distortions in the image generated [110].

Piezoelectric creep is related to the effect of the applied voltage on the remnant polarization of the piezoceramic actuator. If the operating voltage of a piezoelectric actuator is increased, the remnant polarization continues to increase. This manifests itself in a slow creep after the voltage change is complete. A negative step change in the applied voltage would have the opposite effect.

1) Nonlinear Creep Model: A number of approaches have been proposed to deal with this phenomenon. One approach is based on the approximate description of the creep effect by the following nonlinear equation [111]:

$$
y(t)=y_{0}\left\{1+\gamma \log \left(\frac{t}{t_{0}}\right)\right\} .
$$

Here, $t_{0}$ represents the time at which the creep effect is apparent, $y_{0}$ is the value of the actuator displacement at time $t_{0}$, and the creep rate, $\gamma$, is a fixed value, that can be identified by observing the step response of the actuator. The use of such a model to correct for creep effects is illustrated in [36] and [111].

2) Nonlinear Versus Linear Creep Models: A challenge with the nonlinear, creep model (3) is its dependence on the choice of the time-parameter $t_{0}$ used to fit the model [111]. For example, the creep rate $\gamma$ tends to depend on the choice of the time-parameter $t_{0}$. Additionally, for any fixed $t_{0}$, the model is not valid as time $t$ becomes small- the output $y$ becomes unbounded as $t \rightarrow 0$. Similarly, the output becomes unbounded as time $t$ becomes large, i.e., $t \rightarrow \infty$. These modeling difficulties can be alleviated by using a linear creep model [34] discussed in the following.

3) Linear Creep Model: The linear model proposed in [34] captures the low-frequency response of a piezoelectric actuator using creep models applied in mechanics [112]. In particular, the creep effect can be modeled as a series connection of a number of springs and dampers [34], i.e.,

$$
\mathcal{G}_{c}(s)=\frac{1}{k_{0}}+\sum_{i=1}^{N} \frac{1}{c_{i} s+k_{i}}
$$

where $k_{0}$ models the elastic behavior of the actuator at low frequencies, $k_{i}$ is the spring constant, and $c_{i}$ is the damping constant. It has been shown that a model order of three, i.e., $N=3$, would capture the creep effect with reasonable accuracy [34]. An advantage of this frequency-domain approach is that this low-frequency model can be appended to the linear model that describes the vibrational dynamics of the actuator at higher frequencies. This augmented model can then be used to determine a controller to compensate for creep as well as other adverse vibration effects associated with the actuator, as illustrated in [34].
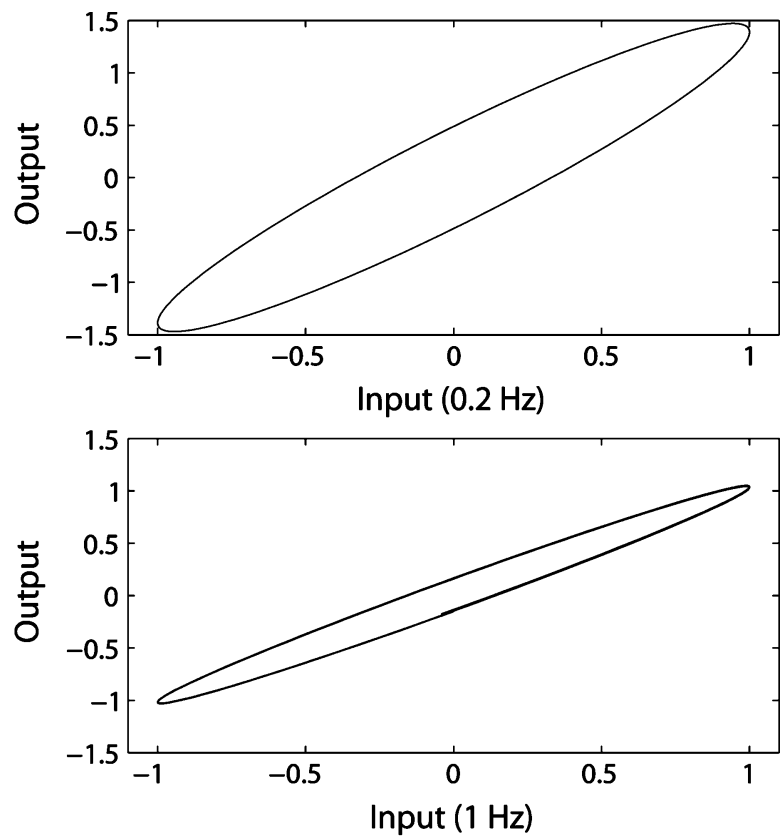

Fig. 14. Steady-state, I/O plots corresponding to phase lag from creep model $\mathcal{G}_{c}$ in (5): (top plot) input is $\sin (0.4 \pi t)$; and (bottom plot) input $\sin (2 \pi t)$ is higher frequency.

\section{B. Hysteresis}

Hysteresis is the main form of nonlinearity in piezoelectric transducers. The original meaning of the word refers to "lagging behind" or "coming after." However, it must not be confused with "phase lag," which is not a nonlinearity and is present in many linear systems. For example, the lag effect of a simplified creep model $\mathcal{G}_{c}$ (in (4) with $N=1, c_{1}=1$ and $k_{1}=1$ )

$$
\mathcal{G}_{c}(s)=1+\frac{1}{s+1}
$$

is shown in Fig. 14 by plotting the input-output signals of the system against each other-these plots change with input frequency. For example, at low frequencies (relative to the poles of $\mathcal{G}_{c}$ ), the creep effect is significant and tends to appear rounded with an overall shape of an ellipse (see Fig. 14, top plot). At higher frequencies, the creep effect reduces and is less rounded (see Fig. 14, bottom plot). This frequency dependence of the input-output ( $\mathrm{I} / \mathrm{O})$ response due to the creep effect is coupled with the hysteresis effect when the I/O plots are generated experimentally. Thus, these two behaviors (creep and hysteresis) are not mutually exclusive; estimation of one behavior's model is influenced by the other behavior [34].

1) Rate and Amplitude Dependence: The level of hysteretic distortion in a piezoelectric actuator will also vary depending on either the maximum value of the input voltage being applied, the frequency of the input signal or both. The last case is referred to as "dynamic" or "rate-dependent" hysteresis [113]-[116]. The hysteresis associated with piezoceramics exhibits nonlocal memory. Hence, the current output state of the actuator not only depends on the current voltage input but also on its past history [117]. This property can cause complications in feedback control systems if it is not accounted for, as several different output states can be obtained from the same input value depending 


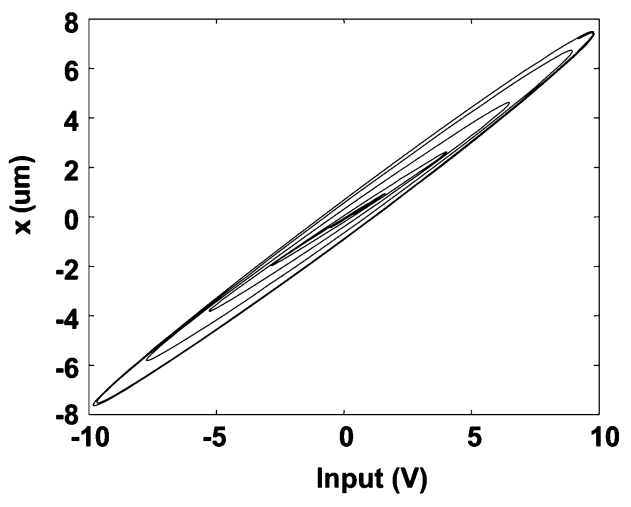

Fig. 15. Example hysteresis loops between input voltage $v$ and the lateral movement $x$ of an experimental piezoelectric tube.

on the "memory" (or past history) stored in the piezoelectric material.

2) Effect on Closed-Loop Stability: The adverse effect of hysteresis on the closed-loop stability and performance of voltage-controlled piezoelectric actuators has been reported by several authors. In particular, [118] reports experimental results to illustrate that when a piezoelectric stack actuator is being used in a feedback loop with a voltage amplifier, the gain and phase margins of the system will deteriorate significantly (up to $28 \%$ ) owing to the presence of hysteresis.

3) Hysteresis Modeling: To successfully exploit the full potential of piezoelectric transducers in control schemes, it is essential to understand and model their behavior accurately. When the output is plotted against the input, the plots for increasing and decreasing inputs are different and form a loop (see Fig. 15); early models used polynomials to capture the major loops in the I/O response, e.g., [119]. However, such approaches fail to capture the minor loops seen in Fig. 15. The most widely used approach to model hysteresis in piezoelectric materials is the classical Preisach hysteresis model [120]-[123]. Another one is the Maxwell resistive capacitor (MRC) model [121], [124]. The connection between the two approaches has also been investigated [125].

The classical Preisach model has been shown to model hysteresis accurately at a given frequency in many different types of actuators, such as electromagnets [126], shape memory alloys [127], ferromagnets [128], and piezoceramics [34]. A practical implementation of the procedure was described in [120].

4) Modeling the Input-Frequency Dependence: Note that the classical Preisach approach is not rate-dependent and can only be used for applications that operate at a single frequency, such as SPM. For applications that require a broadband compensation, a dynamic Preisach model [114]-[116], [123] must be used instead. An alternative approach is to decouple the linear models of creep and vibrations from nonlinear, Preisach models of hysteresis [34]. Specifically, hysteresis is modeled as an input nonlinearity [121] between the applied input voltage $V$ and the resulting mechanical input $F$, as shown in Fig. 16. Next, the output of this hysteresis submodel $F$ is passed through a creep and vibrations submodel [34], which captures the frequency-dependant "phase lag" and "oscillations" in the I/O response that might otherwise be considered as rate dependence (frequency

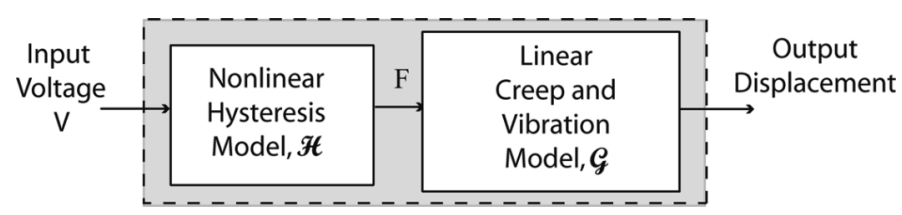

Fig. 16. Schematic of piezoelectric actuator model. For an input $V$, the output is given by $\mathcal{G}[\mathcal{H}(V)]$. The structural vibrations and some of the apparent ratedependence in the hysteresis effect are captured in a linear model $\mathcal{G}$ and the hysteresis nonlinearity $\mathcal{H}$ is captured, using, e.g., a rate-independent Preisach hysteresis model.

dependence) in the hysteresis. Interchanging the order of the linear creep and vibration submodels does not change the inputvoltage-to-output-position response of the model.

5) Charge Control Rather Than Voltage Control: The problem of controlling the hysteresis effect can be substantially eased by the use of charge, or current to drive the piezoelectric actuator. It has been known since the 1980s that the piezoelectric effect in a charge-driven piezoelectric actuator is minimal [129], [130]. However, this unique property of piezoelectric materials has not been widely used because of the difficulties associated with driving highly capacitive loads with available charge/current amplifiers. The main problem is the existence of offset voltages in the charge or current source circuit and the uncontrolled nature of the output voltage, which results in the capacitive load being charged up. When the output voltage, also known as the compliance voltage, reaches the power supply rails, the signal applied to the actuator saturates and distortions occur. This issue has been pointed out by a number of authors [131], [132]. Recent research [32], [133] proposes a new structure for charge and current sources capable of regulating the dc profile of the actuator. A dc-accurate charge/current amplifier has also been proposed in [134] and [135].

\section{Vibrations}

A major obstacle in achieving high-speed nanopositioning in applications such as SPM is the vibrations induced when the positioning bandwidth is increased relative to the first resonant mode of the piezoelectric actuator. This loss of positioning precision is generally small at low operating speeds. Consequently, the operating bandwidth of these systems is restricted to be much smaller than the first resonance frequency of the piezoelectric actuator. In applications such as SPM, this amounts to a scanning speed that is $10-100$ times lower than the first resonance mode of the actuator.

To appreciate the complications that can arise during highspeed nanopositioning applications, note that in SPM a probe is moved over the sample in a raster pattern. To achieve this specific movement of the probe, a slowly increasing ramp signal is applied to the $y$-electrode of a piezoelectric tube scanner, while the $x$-electrode is driven by a fast triangular waveform. When the frequency of this latter waveform is high, the lateral movement of the tube is distorted. This situation is illustrated in Fig. 17(top), which depicts the lateral movement of a piezoelectric tube when driven by a $40-\mathrm{Hz}$ signal. The first resonant mode of the tube is located at $850 \mathrm{~Hz}$. The distortions are due to the amplification of the 21 st and 23rd harmonics of the triangular waveform, which are close to the first resonant mode of 

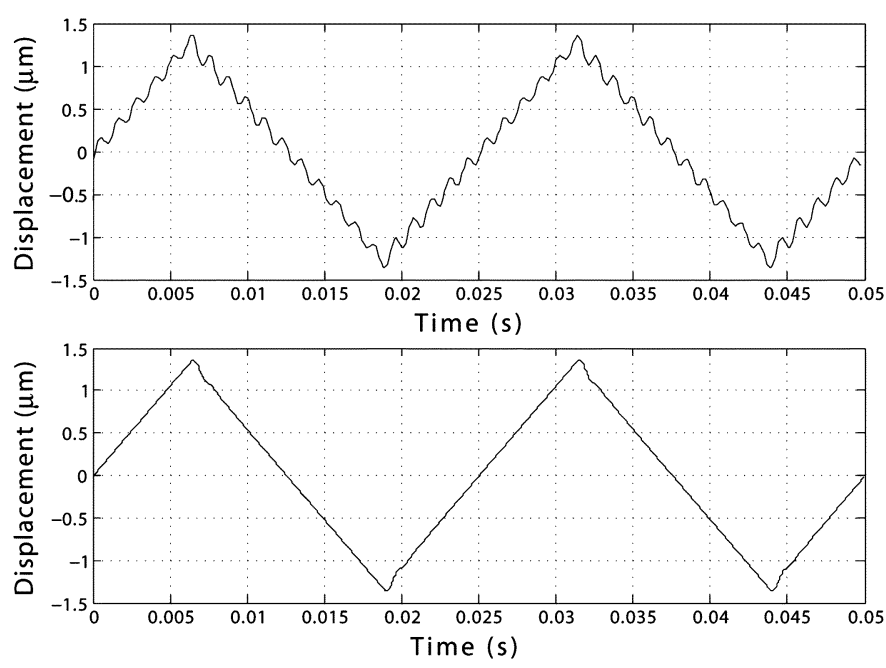

Fig. 17. Open-loop (top) and closed-loop (bottom) lateral movement of an experimental piezoelectric tube when driven by a $40-\mathrm{Hz}$ signal. First resonance frequency of the tube is at $850 \mathrm{~Hz}$. Reprinted from [136] with permission.

the tube. These distortions can be substantially reduced if this resonant mode is damped. Fig. 17(bottom) illustrates the improvement that can be achieved if $25-\mathrm{dB}$ damping is added to the first mode of the structure [136].

\section{Modeling Errors}

The system performance (controller design) should be robust to the presence of modeling errors due to parameter variations and unmodeled dynamics.

1) Parameter Variations: A major difficulty in modeling piezoelectric-actuator dynamics is that parameters (such as the applied-voltage to induced-strain constant and the external load) are not known accurately. Therefore, it is challenging to develop a priori accurate models for controller design. Even when the parameters are known, they can change over relatively long time intervals because of aging effects. Moreover, piezoelectric parameters are very sensitive to variations in temperature [137]. Therefore, experimental modeling and parameter identification are important aspects of the controller design, thus making robust, adaptive, and learning techniques well suited for the control of piezoelectric-actuator-based systems.

2) Unmodeled Dynamics: When designing controllers for the vibrational dynamics of piezoelectric actuators, highfrequency vibrational modes are often neglected to obtain a simplified model (for controller design). However, the high-frequency vibrational modes can affect the stability of the closed-loop system as well as impose limitations on the achievable performance of the closed-loop system. Therefore, the spillover effects on these unmodeled modes should be considered in the controller design [138].

3) Coupling Effects: Another type of unmodeled dynamics is the cross-coupling effect between different axes (e.g., of a piezoelectric tube), which is often assumed to be negligible. However, it has been shown that such effects could become significant if the actuator is used in a high-speed scanning regime [139], [140]. Therefore, there is a need for better understanding cross-

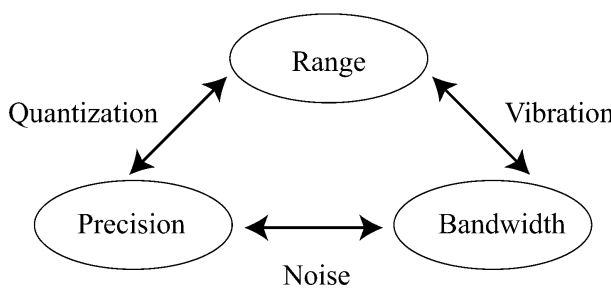

Fig. 18. Tradeoffs between bandwidth, precision, and range.

coupling effects and multiaxis, MIMO controllers in piezoelectric tube scanners.

\section{E. Bandwidth-Precision-Range Tradeoffs}

Controller design has to consider tradeoffs between the bandwidth, precision, and range of a piezoelectric actuator, as shown in Fig. 18. Positioning precision depends inversely on the bandwidth, not only because of the difficulty in controlling higherfrequency modes of the piezoelectric actuator (which leads to a loss in precision), but also because the sensor noise tends to be proportional to the bandwidth. Moreover, precision also depends inversely on the positioning range because of quantization noise in digital controller implementations. For example, analog-to-digital conversion (when using analog sensors such as thermal and capacitive sensors) as well as digital-to-analog conversion (for actuation) introduce noise, which adversely affects the controller performance.

The bandwidth tends to be inversely dependent on the range because the first vibrational resonance of the piezoelectric actuator tends to be higher for a smaller actuator. Note that vibrations tend to degrade positioning accuracy as the main frequency content of the input becomes close to the first resonance frequency of the system. One approach to reduce vibration-induced error is to choose system inputs that avoid exciting the piezoelectric positioner's vibrational dynamics. Specifically, vibrations can be decreased by limiting the input frequency content to well below the system resonance (low-speed operation). Alternatively, to enable higher-speed operation, the first resonance frequency of the system can be increased by optimizing the geometry of the piezoelectric positioner (to make it stiffer). This optimization, however, usually results in a smaller (or stiffer) piezoelectric positioner, which also tends to have a smaller maximum positioning range. Therefore, the tradeoffs are between the maximum range and the achievable bandwidth of the piezoelectric positioner.

\section{CONTROL APPROACHES}

This section reviews the literature on controller development for nanopositioning with piezoelectric actuators. The different approaches are broadly classified into feedback, feedforward, iterative, and sensorless control.

\section{A. Feedback}

1) Integral Controllers: The main advantage of an integral controller is that it provides high-gain feedback at low frequencies; therefore, integral controllers can overcome creep and hysteresis effects and lead to precision positioning (since the vibrational dynamics is not dominant at low frequencies). 


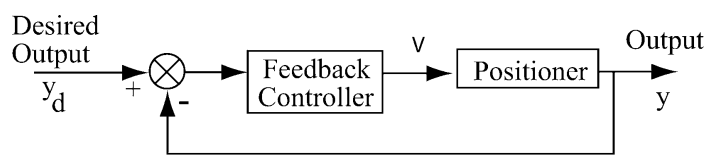

Fig. 19. Feedback control scheme, where the positioner dynamics was modeled in Fig. 16.

In this sense, traditional proportional-integral-derivative (PID) feedback controllers (as shown in Fig. 19), or a double integral for tracking a ramp, are well suited for nanopositioning [141]-[143] and are popular in SPM applications [144]. Recently considerable research effort has gone into the automated tuning of the PID parameters [145] as well as the robustification of existing integral controllers [37].

2) Low Gain Margin: Precision positioning can also be achieved at high frequencies if feedback controller gains can be chosen to be sufficiently high at those frequencies to overcome vibration-induced errors. Nonetheless, there are limits to the improvements achievable in positioning performance with high-gain controllers because piezoelectric actuators tend to have low gain margins. The low gain margin is a result of rapid loss in phase at the sharp resonant peak (i.e., first vibrational resonance) in the frequency response (Bode plots) in combination with loss in phase due to higher-frequency dynamics and filters used with sensors and actuators. Therefore, large feedback gain at high frequency tends to destabilize the piezoscanner [118], [144]. One approach to resolve the low-gain margin problem is to modify the first sharp resonant peak of the system with a notch filter [146], [147]. Experimental results show that this notch-filter approach can lead to a marked increase in the gain margin, and can be used to design feedback controllers that significantly improve the closed-loop performance in piezoelectric actuators-even at high frequencies [148].

3) Modern Control Techniques: The main challenge in feedback design is performance improvement while maintaining the stability of the overall system in the presence of parameter uncertainty and unmodeled high-frequency vibrational modes [138]. Therefore, advanced control techniques have been applied to improve the precision and bandwidth of piezoelectric actuators used in nanopositioning significantly. These control techniques include state feedback [146], adaptive methods [149], [150], and lead/lag methods [101], [151]. Furthermore, robust control techniques have been developed in [35]-[37], [101] and [102].

\section{B. Feedforward}

Use of feedforward can lead to improved output-tracking performance in SPM [33]. Such feedforward usually augments the feedback controller (see Fig. 20) - the ideal feedforward (to achieve perfect tracking of the desired output, $y=y_{d}$ ) is the inverse $\mathcal{H}^{-1}\left[\mathcal{G}^{-1}\left(y_{d}\right)\right]$ of the positioner dynamics, $\mathcal{G}[\mathcal{H}(V)]$ in Fig. 16.

1) Feedforward Hysteresis Compensation: Several methods have been proposed to deal with hysteresis in piezoceramics, most of which are based on feedforward inverse compensation schemes, which invert mathematical models of the hysteresis

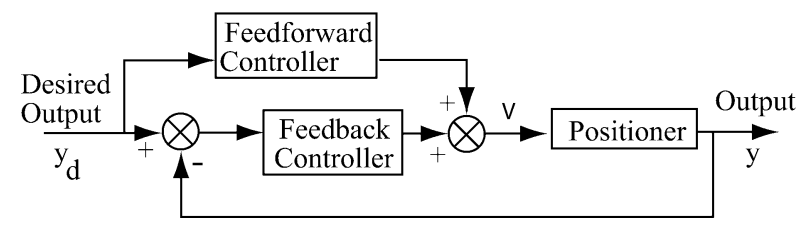

Fig. 20. Augmenting feedback with feedforward.

nonlinearity ( $\mathcal{H}$ in Fig. 16) to determine hysteresis-compensating inputs. Such hysteresis inverse is sufficient during low-frequency operation since creep can be corrected using feedback and vibrations are not significant at low frequencies. The inversion is a two-step process. First the hysteresis $\mathcal{H}$ is fitted with, e.g., polynomials [119], [152], exponentials [153], the Preisach model [117], [126], [154], the MRC model [121], the Prandtle-Ishlinskii model [155], multiple linear-play models [156], differential equation models [157], or deterministic path models [111], [158]. Then, the model is inverted to obtain $\mathcal{H}^{-1}$ in a second step. An alternative approach (which reduces the number of steps) is to directly capture the inverse model $\left(\mathcal{H}^{-1}\right.$, e.g., using a Preisach technique) and use it to find the input signal [34]. In such feedforward methods, the desired output is fed through the inverse model to obtain the input signal, which is then passed onto the physical plant, the output of which will ideally be the desired signal.

The challenge with such approaches is the modeling complexity and parameter sensitivity. Therefore, methods for dealing with hysteresis have been reported that avoid the need for an accurate model. One such method is the phaser approach, reported in [151] and [159], which essentially represents hysteresis as a linear phase lag and attempts to compensate for it by using a phase lead transfer function in series with the plant. Describing functions have also been implemented with some success [118], [131].

2) Increasing the Bandwidth: The feedforward method shown in Fig. 20 does not share the low-gain margin problem of the feedback approach. Therefore, substantial improvements in precision positioning at high frequency can be achieved by using feedforward techniques [160]. This ability to increase the bandwidth with model-based feedforward while achieving subnanometer-scale positioning precision was first demonstrated experimentally for an SPM in [33].

Similar to hysteresis compensation, the feedforward approach uses a mathematical model of the system's linear dynamics ( $\mathcal{G}$ in Fig. 16) to determine vibration-compensating inputs for piezoelectric positioners as shown in [33] - this is augmented with the hysteresis inverse $\mathcal{H}^{-1}$ to invert the entire system model $\mathcal{H}^{-1}\left[\mathcal{G}^{-1}\left(y_{d}\right)\right]$ as shown in [160]. Recent advances in inversion methods (to find the feedforward input) can tradeoff actuator limitations with the required positioning precision [33], [160], [161], exploit actuator redundancy when using multiple actuators [81], enable robust inversion of the actuator dynamics [162]-[164], enable online implementation of the inversion approach [165], and reduce the computational effort [166].

3) Integration of Feedforward and Feedback: Feedforward control improves performance without incurring the stability problems associated with feedback design. However, it cannot 


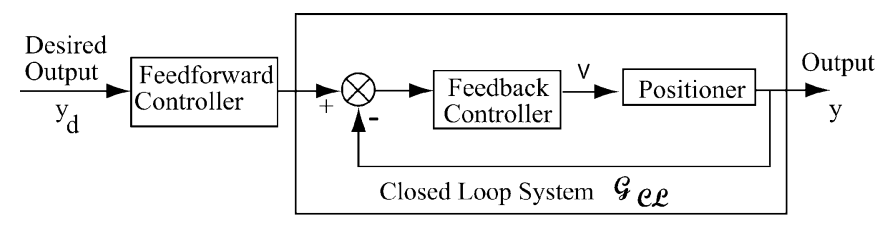

Fig. 21. Inversion-based feedforward of closed-loop system $\mathcal{G}_{\mathcal{C} \mathcal{L}}$. The feedback controller reduces the system nonlinearity and uncertainty; the feedforward is the inverse $\mathcal{G}_{\mathcal{C} \mathcal{L}}^{-1}$ of the linearized closed-loop system.

account for modeling errors. In particular, inversion-based feedforward controllers (which are model-based) cannot correct for tracking errors due to plant uncertainties [167]. Therefore, it is necessary to use feedback in conjunction with feedforward to reduce uncertainty-caused errors in the inverse input. Note that the use of feedforward inputs can improve the tracking performance compared with the use of feedback alone, even in the presence of plant uncertainties. The size of acceptable uncertainties to guarantee that performance can only get better by adding feedforward to feedback has been quantified in [162].

4) Modeling and Computational Complexity: Although the feedforward input obtained using model-based inversion improves positioning precision [34], a challenge in using such feedforward methods is the computational effort required to invert the piezoelectric-actuator dynamics, which include both the nonlinear hysteresis effects and the linear vibrational dynamics. The challenge arises because of the complexity of developing and identifying high-order hysteresis models. Moreover, inversion of these high-order models can be computationally challenging. Inversion of the linear vibrational dynamics $\mathcal{G}$ is relatively simpler [33] than inversion of a nonlinear hysteretic system with vibrations $\mathcal{G}[\mathcal{H}]$ in Fig. 16.

One approach to reduce the modeling and computational complexity is to first linearize the system by using feedback to overcome the hysteresis effects - issues in the use of high-gain feedback for linearization are discussed in [148]. Next, the linearized closed-loop system $\mathcal{G}_{\mathcal{C} L}$ can be inverted [148], [166], which is easier to invert than the complete nonlinear dynamics, to find the feedforward input (see Fig. 21). While such inversion of the closed-loop system leads to reduction in modeling and computational complexity, a drawback of this approach (when compared to the scheme in Fig. 20) is that the achievable positioning bandwidth (of the closed-loop system $\mathcal{G}_{\mathcal{C} L}$ ) could be limited by the low-gain margin problem in the design of the feedback controller.

Alternatively, the system nonlinearity can be reduced by using charge control, and then the inversion-based approach can be applied to the linearized system to obtain the feedforward input [168].

5) Reducing Uncertainty With Feedback: The feedback controller in Fig. 21 can be designed to reduce the uncertainty in the closed-loop system. This reduces the error in modeling the closed-loop system $\mathcal{G}_{\mathcal{C} \mathcal{L}}$, and therefore, reduces the error in computing the inverse input [148], [166]. Again, a drawback of this approach to invert the closed-loop system is that the achievable bandwidth is limited by the low-gain margin problem in the design of the feedback controller. Alternate strategies to reduce uncertainty in the inversion process include: adaptive inversion of the system model (for both schemes, Figs. 20 and 21), see e.g., [169], or learning the correct inverse input that yields perfect output tracking, i.e., iterative inversion of the system model, e.g., [140] and [170].

\section{Iterative Control}

If the positioning application is repetitive (e.g., periodic scanning of the SPM probe), iterative methods can be used to improve the positioning performance. Therefore, iterative and adaptive control methods are well suited for nanopositioning applications.

1) Hysteresis Control: Adaptive and iterative control approaches lead to a reduction in hysteresis effects, and thereby an improvement in positioning precision, e.g., [145], [149], [150], [171], and [172]. A challenge in iterative approaches for hysteretic systems is to prove convergence of the iterative algorithm. The difficulty in proving convergence arises because the output is multivalued for a given input (see Fig. 15). Therefore, the direction in which one needs to change the input (i.e., whether to increase or decrease the input to correct an output error) cannot be uniquely found [173]. The design of iterative control algorithms with guaranteed convergence rates for piezoelectric actuators has been studied in [173]-[175].

2) Vibration Control: Iterative control can significantly increase the operational bandwidth of piezoelectric actuators without loss of positioning precision. For example, in SPM applications, iterative feedforward approaches can be used to position the probe precisely over a sample surface [140], [176]. Such iterative approaches are particularly useful for precise control of the tip-sample interactions such as the tip-sample force to avoid damage of soft biological samples during SPM imaging [140], [176].

\section{Sensorless Control}

The idea of sensorless control of scan-induced vibrations in piezoelectric tube scanners is based on the observation that a piezoelectric transducer can function as an actuator, a sensor, or both simultaneously. Exploiting this embedded sensing capability of piezoelectric transducers eliminates the need for an external sensor in feedback-based vibration control systems.

1) Piezoelectric Tube Scanners: Two concepts for sensorless control are illustrated in Fig. 22. In the first approach, illustrated in Fig. 22(a), one of the electrodes of a piezoelectric tube is used as a sensor, while the other electrode functions as an actuator. The purpose of the controller is to minimize structural vibrations of the tube associated with its first resonant mode by adding sufficient damping to the structure. This is a collocated feedback control system, and a number of control design techniques have been proposed in the literature that are applicable in this case, see, e.g., [177]-[182]. Once sufficient damping has been achieved, tracking can be obtained by driving the system with a scanning signal as shown in Fig. 22(a). An application of this methodology to a piezoelectric tube scanner is reported in [183]. A downside of this approach is that the scanning range would be halved as only one of the electrodes can be used to actuate the tube.

The second concept for sensorless control is illustrated in Fig. 22(b) and is generally referred to as piezoelectric shunt 


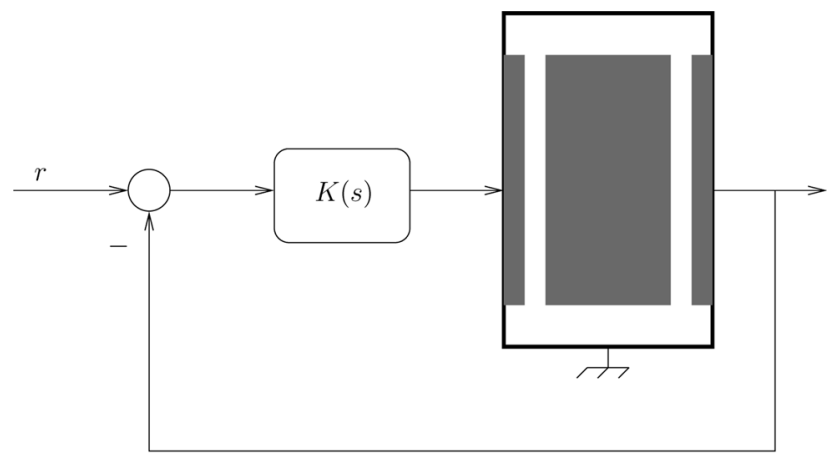

(a)

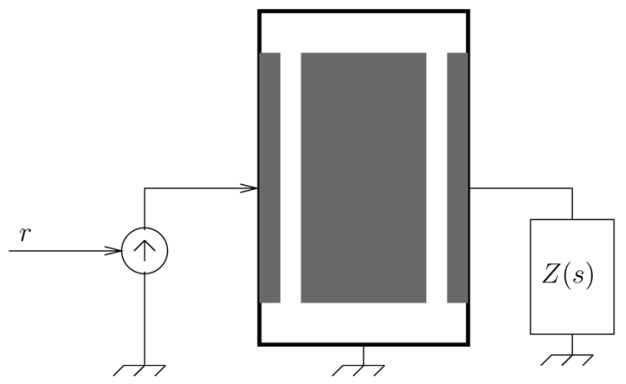

(b)

Fig. 22. Sensorless control of a piezoelectric tube scanner. (a) Strain voltage induced in one electrode is used as the measurement for feedback controller $K(s)$ that drives the opposite electrode. (b) One of the electrodes is electrically shunted to an impedance $Z(s)$.

damping. By shunting one of the electrodes with an appropriate impedance, structural vibration of the tube can be reduced, and the opposite electrode can be used to drive the tube with the scanning signal. Although first described in [184], the concept of piezoelectric shunt damping is usually attributed to Hagood and von Flotow because of their analysis reported in [185]. A series inductor-resistor network has been demonstrated to reduce the magnitude of a single structural mode significantly. Together with the inherent piezoelectric capacitance of the tube, the network is tuned to the resonance frequency of a single structural mode. Similar to a tuned mechanical absorber, additional dynamics introduced by the shunt circuit act to increase the effective structural damping [185].

Piezoelectric shunt control has been the subject of intense research, and interesting results have been reported, see [92], [186], [187], and the references therein. In particular, the invention of synthetic impedance for the digital implementation of an impedance shunt [188] and the identification of the feedback structure associated with shunted piezoelectric transducers [189] have enabled researchers to design and implement highperformance shunts in a very effective way. Application of this concept to a piezoelectric tube nanopositioner is reported in [135]. An advantage of this approach over collocated feedback, illustrated in Fig. 22(a), is that after a slight modification of the shunt impedance the same shunted electrode can be used to drive the actuator with the scanning signal [135]. Therefore, the full scanning range of the nanopositioner can be recovered.

2) Image-Based Sensorless Control: Sensorless control becomes important in subnanometer-scale positioning needed

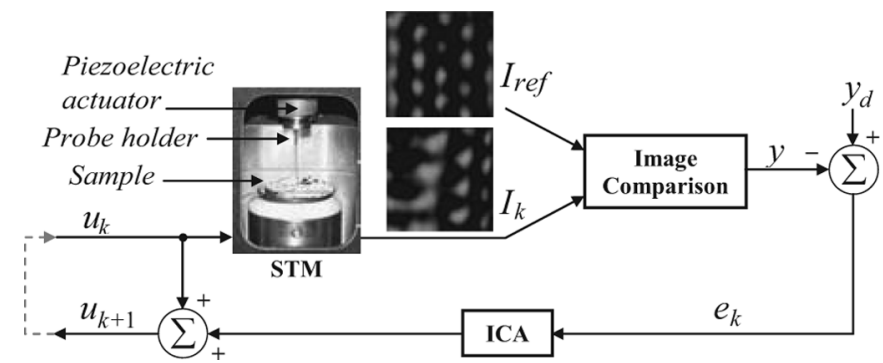

Fig. 23. Image-based STM iterative control [190]. At each iteration step $k$, the STM is used to acquire a reference low-speed and a high-speed image ( $I_{\text {ref }}$ and $I_{k}$, respectively). These two images are compared to determine the positioning error $e_{k}$, which is used by the iterative control algorithm (ICA) to determine the input $u_{k+1}$ for the next iteration step to improve the STM's positioning accuracy. (Image provided by Garrett Clayton.)

when imaging surfaces at the atomic scale with a scanning tunneling microscope (STM). One of the difficulties with feedback approaches in STM is that external sensors cannot directly measure the position of an STM-probe's atomically sharp tip. Instead, they measure the position of a different point on the STM scanner and then infer the position of the STM-probe's tip. Thus, it is challenging to directly measure the position of the STM-probe's tip over the sample surface with external sensors, also making feedback control challenging.

Moreover, the resolution of conventional sensors is not sufficient for feedback control of the STM when subnanometer resolution is needed at high speeds (at room temperature) because sensor noise tends to increase with the scan frequency and temperature. For example, while the resolution of noncontact capacitive sensors is only limited by quantum noise (in theory), the effective noise factor is about $0.01 \mathrm{~nm} / \sqrt{\mathrm{Hz}}$. Therefore, the resolution becomes poor during high-speed STM operation; the resolution is about $0.1 \mathrm{~nm}$ at $100 \mathrm{~Hz}$, which is not sufficient to correct for positioning errors when imaging a few carbon atoms in graphite, where the spacing of the atoms is approximately $0.2 \mathrm{~nm}$. This lack of high-resolution measurement capabilities makes the use of feedback control to compensate for dynamic effects in STMs challenging.

To resolve problems with using external sensors, an imagebased approach (see Fig. 23) was developed that exploits the extant imaging capability of the STM in [190]. This approach, which uses image distortions to compensate for dynamic effects, extends previously developed methods that have used STM images to correct for positioning errors at relatively low operating speeds [191]. The main idea is to quantify the error in positioning the STM-probe's tip over the sample surface by using STM images of standard calibration samples. As the calibration sample surface is fixed, distortions in the image (due to dynamics effects) can be used to quantify the positioning errors and correct the input to the STM. In general samples (rather than calibration samples), topography-feature recognition can be used to correct dynamic effects (e.g., to correct creep effects [192]). Thus, the ability to quantify and correct dynamic effects is only limited by the resolution of the built-in, tunneling-current sensor (of the STM) and not by limitations of external position sensors. Also, because an image-based approach exploits 
the extant imaging capability of the STM, its use enables an increased operating speed without requiring additional hardware, and, therefore, without substantially increasing equipment cost.

An advantage of the image-based approach is that it can be automated using image-distortion-based error estimation algorithms such as those developed in the visual-servoing field (see, for example, [193]). Such automation will make it easy for the end user to not only calibrate, but also recalibrate the STM to account for variations in the scanner dynamics caused by time-varying effects (e.g., aging-related effects) and operating conditions (e.g., temperature). Automation will also allow the image-based approach to be applied to highly parallel microfabricated STM systems that are being developed for nanotechnologies. Such miniaturized arrays have higher bandwidth [194] and increased throughput [195], but their operational speed is still limited by dynamic effects. Thus, higher-speed operation of such miniaturized parallel systems can also be enabled by exploiting the image-based approach.

\section{EMERGING CONTROL IsSUES}

In this section, we will discuss some of the emerging applications and control issues in nanopositioning.

\section{A. Precision Positioning Over Long Range}

The need for positioning systems with nanometer-scale precision over extended ranges has typically been given a lower priority on the basis that nanotechnologies (such as nanofabrication) were purely limited to research [196]. However, longrange high-precision positioning is becoming increasingly critical as we realize the many possibilities nanotechnology has to offer. For example, such positioning systems are particularly important to bridge the gap between micro- and nanofabrication when linking compound geometric patterns containing nanostructures with their much larger I/O connections [196]. Current commercially available inchworm motors offer both high precision and long range-they are capable of nanometer-precision positioning over the centimeter range (see, e.g., [26], [27], [197], and [198]). However, because of vibrations induced during locomotion, the positioning precision of inchworm motors is lost during high-speed operation.

1) Long-Range High-Precision Positioning With Inchworm Motors: Inchworm motors are capable of delivering high precision over long-range motions. As shown in Fig. 24, the piezoelectric motor is stationary (fixed to the ground at $\mathrm{C}$ ) while the inner rod travels back and forth. (A kinematic variation is to hold the rod stationary and let the inchworm motor move over the rod.) In such motors, relatively large movements with nanometer-scale precision can be achieved by repeating a nanostepping procedure. Briefly, the substeps involved in a nanostep movement are as follows (see Fig. 24). First, piezoelectric actuator A clamps to the rod; then piezoelectric actuator $\mathrm{B}$ expands, forcing the rod to move to the left; piezoelectric actuator C clamps and A releases, and finally, B contracts, forcing the rod to move to the left again. This motion, repeated over and over again, allows the inchworm motor to achieve nanometer-precision positioning of the rod over a long range.
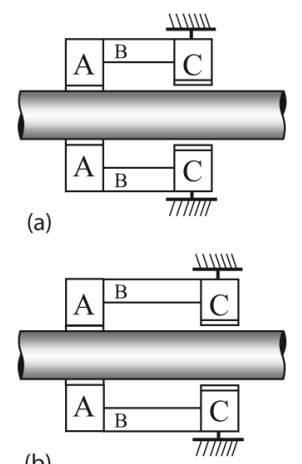

(b)

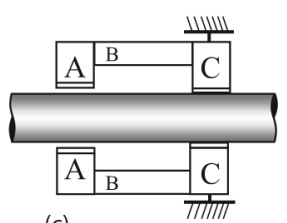

(c)

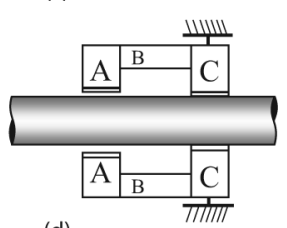

(d)
Fig. 24. Schematic of an inchworm moving a rod to the left-this requires repeated clamping and unclamping of piezoelectric actuators A and C. (a) A clamps to the rod, (b) B expands, forcing the rod to move to the left, (c) C clamps and A releases, and (d) B contracts, again forcing the rod to move to the left.

2) Impact Control in Inchworm Stages: Impact-induced vibrations that result from each clamping step (piezoelectric actuators $\mathrm{A}$ and $\mathrm{C}$ ) become more and more pronounced with increasing operating speed, and lead to loss of positioning precision during high-speed operation. Furthermore, each on-off cycle of a clamp produces dynamic tensile stresses that will ultimately produce cracks in the piezoelectric material. For example, the fatigue life of piezoelectric actuators in the inchworm motor (used by Burleigh Instruments, Inc.) ranges from 100 million to one billion clamp-change cycles. If the inchworm motor takes nanometer-sized steps, its estimated life is on the order of 200 to $2000 \mathrm{~m}$. This estimated life is extremely low if such a device is to be used for long-range positioning (with nanometer precision). A typical recommendation to reduce the possibility of fatigue failure of the piezoelectric actuator (at present) is to avoid mechanical shocks-i.e., to go slow. Therefore, control techniques are needed to mitigate the adverse effects of impact forces and vibration during rapid positioning with inchworm motors.

\section{B. Increasing Throughput}

The throughput of current SPM-based nanofabrication is too low to be economically viable. Therefore, a substantial increase of the throughput is needed.

1) Use of SPM in Nanofabrication: Although SPM was initially developed and applied — with tremendous success—as a surface analysis instrument, its potential for surface and material modification is now being exploited for nanofabrication [15]. The main advantage of SPM-based nanofabrication is that it achieves the smallest features [15]: For example, STM achieves the manipulation of single atoms, although in highly restrictive environments [199]. In general, STM can be used for additive nanofabrication by depositing material from a gas precursor (a localized chemical vapor deposition technique) or from an electrolyte (localized electrodeposition). Material can also be removed from the substrate by mechanical means such as contacting the tip against the sample as in AFM or by electrical pulses. Other approaches, such as localized etching of the surface with a gas or electrolyte, also fall under the second category, where material is removed from the substrate. Thus, SPM-based 
techniques are poised to play a significant role in future technological progress, especially when manufacturing processes in electronics, optics, and precision machining begin to require nanometer-scale control over feature sizes.

2) Low Throughput of SPM-Based Nanofabrication: Unfortunately, SPM-based nanofabrication suffers from throughput limitations present in all serial techniques-the tip must visit each point where something is to be done. Even with multiple probes [195], [200] such serial processes cannot compete with parallel techniques like current optical lithography, which can process an entire wafer (more precisely, one die) in one step.

3) Integrating Top-Down and Bottom-Up Nanofabrication: One solution to the low-throughput problem is to integrate the slower, top-down, SPM nanofabrication with faster, bottom-up, nanofabrication methods. For example, rather than adding all the required material in a direct write approach, STM-based chemical vapor deposition (CVD) might be used only for seeding or prenucleating the desired pattern, whereas the rest of the material can then be grown by selective CVD [201]. Similarly, patterned self-assembled monolayers can be fabricated with AFM-based dip-pen nanolithography, which can then be used for nucleation and growth of functional polymers [202], [203]. In this sense, the top-down SPM is only needed for generating the initial pattern, which then forms the basis for growing the nanostructure using highly-parallel bottom-up techniques [204], [205].

4) Need for High-Speed Positioning: The generation of initial seed patterns with an SPM will require rapid positioning of the SPM probe (i.e., the output) from one location to another on the initial seed pattern. Therefore, optimal control techniques and trajectory-planning methods are well suited to rapidly shift the SPM probe from one position to another [206], [207]. Similar methods have also been used to optimize the scan paths in SPMs [208].

\section{Distributed Nanopositioning Systems}

Distributed arrays of nanopositioners lead to highly parallel systems, which have both long range as well as high bandwidth. Control of such distributed systems is an emerging area in applications such as data storage and nanofabrication.

1) Storage Application: The ability to modify surfaces with an SPM has motivated efforts to create data-storage devices based on scanning-probe techniques for storing data at much higher densities than can be achieved by conventional means, e.g., [30] and [209]-[211]. These devices are still based on mechanical access, but MEMS-based actuators, sensors, and micro-scanners will replace the microscopic actuators, motors, and mechanical structures of conventional storage devices. It is the unique capabilities of the MEMS structures that promise small form factor, low power consumption, fast mechanical access times, and good shock robustness. In such storage devices, the rotating disk and single head per surface paradigms of HDD have been abandoned in favor of MEMS-based $x / y$ actuators (in some designs $z$-direction actuation is necessary) that position a large 2-D array of probe tips over the storage medium for parallel READ/WRITE operations. The schematic illustration of an example device is shown in Fig. 25. As actuation distances are typically on the order of $100 \mu \mathrm{m}$, and

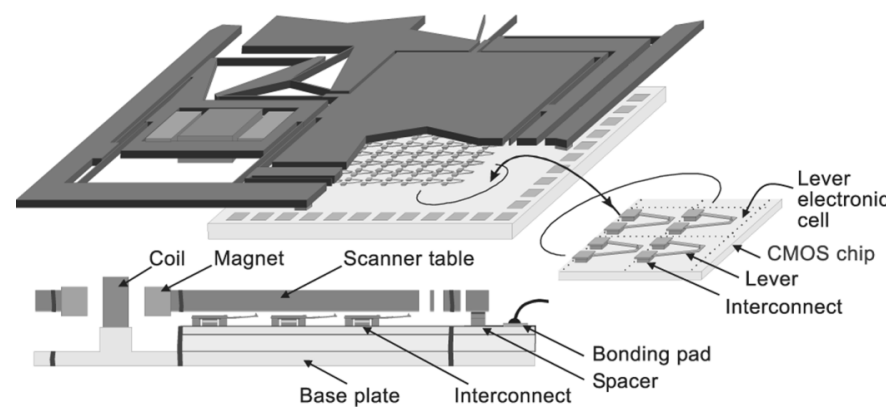

Fig. 25. Schematic of MEMS-based probe-storage device. (Reprinted with permission from [212].)

components have small masses, the positioning delays, i.e., access times, are much smaller than in disk drives. However, to achieve the ultrahigh storage densities that are possible with scanning probes, extreme precision in navigating the probe tips to the desired position is required.

2) Design Requirements: Functional storage devices require mechanical motion between the READ/WRITE probes and the storage medium. This is accomplished by a microscanner consisting of a movable table on which the medium (or alternatively, the probe array) is located, a spring system with sufficient compliance to accommodate the required amount of motion, and an actuation means with sufficient force to move the table the desired distance and with the desired acceleration. Although conceptually such a microscanner is a simple device, the design requirements are quite stringent, and include not only an independent and orthogonal $x-y$ travel range of 50 to $200 \mu \mathrm{m}$, high repeatability and linearity, and undesirable out-of-plane motion of less than a small fraction of a micrometer, but also an actuation bandwidth that is sufficient to accommodate a seek/ settle time of less than $1 \mathrm{~ms}$. Moreover, such a microscanner should have a small size that is suitable for flashcard form factors, shock, and vibration resistance-both operational (typically $5 \mathrm{~g}$ ) and nonoperational (survive up to $2000 \mathrm{~g}$ or more), low power consumption, low driving voltage, and low cost. In addition, some microscanner designs also require $z$-axis and tilt-correction actuation.

3) Types of Distributed Actuators: A wide range of concepts have been proposed for storage devices, including electromagnetic scanners [73], [213], [214], scanners with an electrostatic interdigitated comb drive [66] or a stepper-like electrostatic surface drive [67], and an electrostatic clamp-and-slip design [71], [215]. Notably absent is a piezoelectric design: Although piezoelectric actuators are in widespread use in SPMs, it is difficult to generate the desired stroke in the small space available in a microscanner.

To achieve as low a driving force and power consumption as possible, the table mass and the $x-y$ spring stiffness should be minimized. On the other hand, high $z$-stiffness is required unless the design also includes $z$-axis actuation. Therefore, high-aspect-ratio (as high as 40:1) cantilever springs are used, which are commonly formed by means of DRIE of Si [216]. Constructing the entire scanner from single-crystal Si eliminates drifts and deflections due to thermal mismatches. Moreover, Si springs also have very long life with minimal aging effects due to material 
fatigue, provided they are not overstressed. If narrow springs of approximately $10 \mu \mathrm{m}$ in width are used than a scanner-table travel range of less than $10 \%$ of the spring length will not only prevent overstress, but also result in a highly linear displacement versus actuation force [69], [74].

A MEMS-actuated magnetic probe-based storage system is described in [66] and the references therein: a magnetic storage medium is positioned in the plane, and writing is achieved magnetically by using an array of probe tips, each tip being actuated in the $z$-direction. In [217], an atomic-resolution storage concept is described in which electron field emitters are employed to change the state of a phase-change medium in a bit-wise fashion. One of the most advanced approaches in probe-storage is based on a thermomechanical READ/WRITE process on thin polymer films [210], [211].

4) Need for Nanopositioning Control: Distributed probe storage requires the control of nanometer-scale distances between tracks and recorded marks. For example, in topography-based recording, where the recorded information corresponds to the presence or absence of an indentation (thermomechanical storage on polymers), the typical indentation resembles an inverted cone with nanometer-scale diameter (of approximately 15 to $30 \mathrm{~nm}$ ). Nanopositioning is crucial because the error rate performance deteriorates very rapidly as the distance between the probe and the center of the inverted cone increases (by a few nanometers). In particular, the error rate was found to increase by one order of magnitude at a distance of $4 \mathrm{~nm}$ from the indentation center [218]. This deterioration of offtrack performance is characteristic of probe storage with nanometer-sharp probe tips (used as both WRITE and READ transducers), highlighting the importance of nanopositioning control for distributed systems.

5) Control Issues in Distributed Nanopositioning Systems: Control of distributed nanopositioning systems would require on-chip control schemes. Issues in such control include: developing and implementing embedded, distributed, analog controllers (needed due to fast time scales); managing sensor/actuator failures; and maintaining robustness under substantial uncertainty prevalent in nanoscale phenomena [1].

\section{CONCLUSION}

Nanotechnology is an emerging field, which is opening up novel and challenging problems in control. This paper reviewed some of the control-related research in nanopositioning with piezoelectric actuators and discussed issues in control such as creep, hysteresis, and vibrations. The alleviation of these issues by using different control schemes such as feedback, feedforward, iteration, and sensorless methods were identified. This paper also presented current and emerging applications that require precision control with nanometer-scale positioning.

\section{ACKNOWLEDGMENT}

One of the authors, E. Eleftheriou, would like to thank the entire ZRL probe-storage team, in particular, C. Hagleitner, M. Lantz, and A. Sebastian, for providing detailed comments and suggestions on an earlier draft of this paper. Author S. O. Reza Moheimani would like to thank the Australian
Research Council and the ARC Centre for Complex Dynamic Systems and Control for supporting the research which underlines his contributions to this work.

\section{REFERENCES}

[1] B. Shapiro, "Workshop on control of micro- and nano-scale systems," IEEE Control Syst. Mag., vol. 25, no. 2, pp. 82-88, Apr. 2005.

[2] T. Sheetz, J. Vidal, T. D. Pearson, and K. Lozano, "Nanotechnology: Awareness and societal concerns," Technol. Soc., vol. 27, no. 3, pp. 329-345, 2005.

[3] B. Bushan, Ed., Springer Handbook of Nanotechnology. Berlin, Germany: Springer-Verlag, 2004.

[4] B. C. Crandall, Nanotechnology: Molecular Speculation on Global Abundance. Cambridge, MA: MIT Press, 1996.

[5] G. Binnig and H. Rohrer, "Scanning tunneling microscopy," Helvetica Phys. Acta, vol. 55, pp. 726-735, 1982.

[6] G. Binnig, C. F. Quate, and C. Gerber, "Atomic force microscope," Phys. Rev. Lett., vol. 56, no. 9, pp. 930-933, Mar. 1986.

[7] Q. Zou, K. K. Leang, E. Sadoun, M. J. Reed, and S. Devasia, "Control issues in high-speed AFM for biological applications: Collagen imaging example," Asian J. Control: Special Issue Adv. Nano-Technol. Control, vol. 6, no. 2, pp. 164-178, Jun. 2004.

[8] R. Kassies, K. O. Van der Werf, A. Lenferink, C. N. Hunter, J. D. Olsen, V. Subramaniam, and C. Otto, "Combined AFM and confocal fluorescence microscope for applications in bio-nanotechnology," $J$. Microscopy-Oxford, vol. 217, pt. 1, pp. 109-116, Jan. 2005.

[9] K. D. Jandt, M. Finke, and P. Cacciafesta, "Aspects of the physical chemistry of polymers, biomaterials and mineralised tissues investigated with atomic force microscopy (AFM)," Colloids Surfaces B-Biointerfaces, vol. 19, no. 4, pp. 301-314, Dec. 2000.

[10] I. Schmitz, M. Schreiner, G. Friedbacher, and M. Grasserbauer, "Phase imaging as an extension to tapping mode AFM for the identification of material properties on humidity-sensitive surfaces," Appl. Surface Sci., vol. 115, no. 2, pp. 190-198, Jun. 1997.

[11] K. Yamanaka, A. Noguchi, T. Tsuji, T. Koike, and T. Goto, "Quantitative material characterization by ultrasonic AFM," Surface Interface Anal., vol. 27, no. 5-6, pp. 600-606, May/Jun. 1999.

[12] M. Tsukada, N. Kobayashi, M. Brandbyge, and S. Nakanishi, "Physics of artificial nano-structures on surfaces," Progr. Surface Sci., vol. 64, no. 3-8, pp. 139-155, Jun. 2000.

[13] M. Yves, Scanning Probe Microscopes. Bellingham, WA: SPIE, 1995.

[14] R. Wiesendanger, Ed., Scanning Probe Microscopy and Spectroscopy. Cambridge, U.K.: Cambridge Univ. Press, 1994.

[15] M. Gentili, C. Giovannella, and S. Selci, Eds., Nanolithography: A Borderland Between STM, EB, IB and X-Ray Lithographies, NATO ASI Series E: Applied Science. Norwell, MA: Kluwer, 1993, vol. 264.

[16] P. Vettiger, U. Staufer, and D. P. Kern, Eds., Special Issue Nanotechnol., J. Microelectron. Eng., vol. 32, pp. 1-4, Sep. 1996.

[17] L. R. Harriot, "Limits of lithography," Proc. IEEE, vol. 89, no. 3, pp. 366-374, Mar. 2001.

[18] D. L. White and O. R. Wood, "Novel alignment system for imprint lithography,” J. Vacuum Sci. Technol. B, vol. 18, no. 6, pp. 3552-3556, May/Jun. 2000.

[19] S. Verma, W.-J. Kim, and H. Shakir, "Multi-axis Maglev nanopositioner for precision manufacturing and manipulation applications," IEEE Trans. Ind. Appl., vol. 41, no. 5, pp. 1159-1167, Sep./Oct. 2005.

[20] B. Bushan, Ed., Handbook of Micro/Nano Tribology. Boca Raton, FL: CRC, 1995 .

[21] S. V. Kalinin and D. A. Bonnell, "Effect of phase transition on the surface potential of the $\mathrm{BaTiO}_{3}$," J. Appl. Phys., vol. 87, no. 8, pp. 3950-3957, 2000.

[22] G. M. Whitesides and H. C. Love, "The art of building small," Scientif. Amer., vol. 285, no. 3, pp. 39-47, Sep. 2001.

[23] D. R. Meldrum, W. H. Pence, S. E. Moody, D. L. Cunningham, M. Holl, P. J. Wiktor, M. Saini, M. P. Moore, L.-S. Jang, M. Kidd, C. Fisher, and A. Cookson, "Automated, integrated modules for fluid handling, thermal cycling and purification of DNA samples for high throughput sequencing and analysis," in Proc. IEEE/ASME Int. Conf. Adv. Intell. Mechatron., 2001, pp. 1211-1219.

[24] Z. Rihong, X. Daocai, Y. Zhixing, and C. Jinbang, "Research on systems for measurements of CCD parameters," in Proc. SPIE Detectors, Focal Plane Arrays, Imaging Devices II, 1998, pp. 297-301. 
[25] D. Krogmann et al., "Image multiplexing system on the base of piezoelectrically driven silicon microlens arrays," in Proc. 3rd Int. Conf. Micro Opto Electro Mechan. Syst. (MOEMS), 1999, pp. 178-185.

[26] J. C. Fasik, "An inchworm actuator for the next generation space telescope," Burleigh Instruments, Inc., Fishers, NY, 1998.

[27] B. Zhang and Z. Zhu, "Developing a linear piezomotor with nanometer resolution and high stiffness," IEEE/ASME Trans. Mechatron., vol. 2, no. 1, pp. 22-29, Mar. 1997.

[28] M. Jianxu and M. H. Ang, Jr., "High-bandwidth macro/microactuation for hard disk drive," in Proc. SPIE, Microrobot. Microassembly II, 2000, pp. 94-102.

[29] R. Horowitz et al., "Microactuators for dual-stage servo systems in magnetic disk files," in Springer Handbook of Nanotechnology, B. Bhushan, Ed. Berlin, Germany: Springer-Verlag, 2004, ch. 31, pp. 921-950.

[30] S. Hosaka, A. Kikikawa, H. Koyanagi, T. Shintani, M. Miyamoto, K. Nakamura, and E. Etoh, "SPM-based data storage for ultrahigh density recording," Nanotechnol., Special Issue Scanning Probe-Based Nanoelectron., vol. 8, no. 3A, pp. A58-A62, Sep. 1997.

[31] A. Sebastian, A. Pantazi, G. Cherubini, E. Eleftheriou, M. Lantz, and H. Pozidis, "Nanopositioning for probe storage," in Proc. Amer. Control Conf., 2005, pp. 4181-4186.

[32] A. J. Fleming and S. O. R. Moheimani, "Precision current and charge amplifiers for driving highly capacitive piezoelectric loads," Electron. Lett., vol. 39, no. 3, pp. 282-284, Feb. 2003.

[33] D. Croft and S. Devasia, "Vibration compensation for high speed scanning tunneling microscopy," Rev. Sci. Instrum., vol. 70, no. 12, pp. 4600-4605, Dec. 1999.

[34] D. Croft, G. Shed, and S. Devasia, "Creep, hysteresis, and vibration compensation for piezoactuators: Atomic force microscopy application," ASME J. Dyn. Syst. Control, vol. 123, no. 1, pp. 35-43, Mar. 2001.

[35] G. Schitter, P. Menold, H. F. Knapp, F. Allgower, and A. Stemmer, "High performance feedback for fast scanning atomic force microscopes," Rev. Scientific Instrum., vol. 72, no. 8, pp. 3320-3327, 2001.

[36] S. Salapaka, A. Sebastian, J. P. Cleveland, and M. V. Salapaka, "High bandwidth nano-positioner: A robust control approach," Rev. Scientific Instrum., vol. 73, no. 9, pp. 3232-3241, Sep. 2002.

[37] A. Sebastian and S. M. Salapaka, "Design methodologies for robust nano-positioning," IEEE Trans. Control Syst. Technol., vol. 13, no. 6, pp. 868-876, Nov. 2005.

[38] O. M. El Rifai and K. Youcef-Tomi, "Design and control of atomic force microscopes," in Proc. Amer. Control Conf., 2001, pp. 3251-3255.

[39] A. Stemmer, G. Schitter, J. M. Rieber, and F. Allgoewer, "Control strategies towards faster quantitative imaging in atomic microscopy," Eur. J. Control, vol. 11, pp. 384-395, 2005.

[40] S. Alexander, L. Hellemans, O. Marti, J. Schneir, V. Elings, P. K. Hansma, M. Longmire, and J. Gurley, "An atomic-resolution atomicforce microscope implemented using an optical lever," J. Appl. Phys., vol. 65 , no. 1, pp. 164-167, 1989.

[41] D. Rugar and P. Hansma, "Atomic force microscopy," Phys. Today, vol. 43, no. 10, pp. 23-30, Oct. 1990.

[42] H. G. Hansma, "Varieties of imaging with scanning probe microscopy," in Proc. Nat. Academy USA, 1999, vol. 96, no. 26, pp. 14678-14680.

[43] J. A. Sidles, J. L. Garbini, K. J. Bruland, D. Rugar, O. Zuger, S. Hoen, and C. S. Yannoni, "Magnetic-resonance force microscopy," Rev. Modern Phys., vol. 67, no. 1, pp. 249-265, Jan. 1995.

[44] D. K. Miu and S. P. Bhat, "Minimum power and minimum jerk position control and its applications in computer disk drives," IEEE Trans. Magn., vol. 27, no. 6, pp. 4471-4475, Nov. 1991.

[45] H. T. Ho, "Fast servo bang-bang seek control," IEEE Trans. Magn., vol. 33, no. 6, pp. 4522-4527, Apr. 1997.

[46] S. K. Aggarwal, D. A. Horsley, R. Horowitz, and A. P. Pisano, "Micro-actuators for high density disk drives," in Proc. ACC, 1997, pp. 3979-3984.

[47] K. Mori, T. Munemoto, H. Otsuki, and Y. Yamaguchi et al., "A dual stage magnetic disk drive actuator using a piezoelectric device for a high track density," IEEE Trans. Magn., vol. 27, no. 6, pp. 5298-5300, Jun. 1991.

[48] Y. Li, "Dual-stage servo control and active vibration compensation in magnetic hard disk drives," Ph.D. dissertation, Dept. Mechan. Eng., Univ. California, Berkeley, 2003.
[49] M. Kobayashi and R. Horowitz, "Track seek control for hard disk dualstage servo systems," IEEE Trans. Magn., vol. 37, no. 2, pp. 949-954, Mar. 2001.

[50] Y.-H. Kim and S.-H. Lee, "An approach to dual-stage servo design in computer disk drives," IEEE Trans. Control Syst. Technol., vol. 12, no. 1, pp. 12-20, Jan. 2004.

[51] T. Hirano, H. Yang, S. Pattanaik, M. White, and S. Arya, "A microactuator for tracking servo," in Proc. STLE ASME Joint Tribology Conf. -Symp. Frontiers Magn. Hard Disk Drive Tribol. Technol., 2003, pp. 21-25.

[52] R. B. Evans, J. S. Griesbach, and W. C. Messner, "Piezoelectric microactuator for dual stage control," IEEE Trans. Magn., vol. 35, no. 2, pp. 977-982, Mar. 1999.

[53] M. Tokuyama, T. Shimizu, H. Masuda, S. Nakamura, M. Hanya, O. Iriuchijima, and J. Soga, "Development of a $\phi$-shaped actuated suspension for 100-k TPI hard disk drives," IEEE Trans. Magn., vol. 37, no. 4, pp. 1884-1886, Jul. 2001.

[54] T. Hirano, L.-S. Fan, T. Semba, W. Y. Lee, J. Hong, S. Pattanaik, P. Webb, W.-H. Juan, and S. Chan, "High-bandwidth HDD tracking servo by a moving-slider micro-actuator," IEEE Trans. Magn., vol. 35, no. 5 , pp. 3670-3672, Sep. 1999.

[55] D. A. Horsley, R. Horowitz, and A. P. Pisano, "Microfabricated electrostatic actuators for hard disk drives," IEEE/ASME Trans. Mechatron., vol. 3, no. 3, pp. 175-183, Sep. 1998.

[56] T. Imamura, M. Katayama, Y. Ikegawa, T. Ohwe, R. Koishi, and T. Koshikawa, "MEMS-based integrated head/actuator/slider for hard disk drives," IEEE/ASME Trans. Mechatron., vol. 3, no. 3, pp. 166-174, Sep. 1998.

[57] J. McCormick and R. Horowitz, "Time optimal seek trajectories for a dual stage optical disk drive actuator," ASME J. Dyn. Syst. Meas. Control, vol. 113, no. 3, pp. 534-536, 1991.

[58] J. Ding, F. Marcassa, and M. Tomizuka, "Short seeking control with minimum jerk trajectories for dual actuator hard disk drive systems," in Proc. Amer. Control Conf., 2004, pp. 529-534.

[59] D. Iamratanakul, B. Jordan, K. K. Leang, and S. Devasia, "Optimal output transitions for dual-stage systems," IEEE Trans. Control Syst. Technol., to be published.

[60] W. P. Mason, "Piezoelectricity, its history an applications," J. Acoust. Soc. Amer., vol. 70, no. 6, pp. 1561-1566, 1981.

[61] A. Ballato, "Piezoelectricity: History and new thrusts," in Proc. IEEE Ultrason. Symp., 1996, no. 1, pp. 575-583.

[62] T. Ueno, J. Qiu, and J. Tani, "Magnetic force control with composite of giant magnetostrictive and piezoelectric materials," IEEE Trans. Magn., vol. 39, no. 6, pp. 3534-3540, Nov. 2003.

[63] J. M. Nealis and R. C. Smith, "An adaptive control method for magnetostrictive transducers with hysteresis," in Proc. 40th IEEE Conf. Dec. Control, 2001, pp. 4260-4265.

[64] X. Tan and J. S. Baras, "Modeling and control of a magnetostrictive actuator," in Proc. 41st IEEE Conf. Dec. Control, 2002, pp. 866-872.

[65] S. Verma, W. J. Kim, and J. Gu, "Six-axis nanopositioning device with precision magnetic levitation technology," IEEE-ASME Trans. Mechatron., vol. 9, no. 2, pp. 384-391, Jun. 2004.

[66] L. R. Carley, J. A. Bain, G. K. Fedder, D. W. Greve, D. F. Guillou, M. S. C. Lu, T. Mukherjee, S. Santhanam, L. Abelmann, and S. Min, "Singlechip computers with microelectromechanical systems-based magnetic memory," J. Appl. Phys., vol. 87, no. 9, pp. 6680-6685, 2000.

[67] S. Hoen, P. Merchant, G. Koke, and J. Williams, "Electrostatic surface drives: Theoretical considerations and fabrication," in Proc. 9th Int. Conf. Solid-State Sensors Actuators (Transducers), 1997, pp. 41-44.

[68] S. Hoen, Q. Bai, J. A. Harley, D. A. Horsley, F. Matta, T. Verhoeven, J. Williams, and K. R. Williams, "A high-performance dipole surface drive for large travel and force," in Tech. Dig. (Transducers), 2003, pp. 344-347.

[69] T. Albrecht, M. Despont, E. Eleftheriou, J. U. Bu, and T. Hirano, "MEMS in mass storage systems," in Enabling Technologies for MEMS and Nanodevices, H. Baltes, O. Brand, G. K. Fedder, C. Hierold, J. G. Korvink, and O. Tabata, Eds. Weinheim, Germany: Wiley, 2004, vol. 1, pp. 193-236, ch.6.

[70] L. Abelmann, T. Bolhuis, A. M. Hoesum, G. J. M. Krijnen, and J. C. Lodder, "Large capacity probe recording using storage robots," Proc. IEE Sci. Meas. Technol., vol. 150, no. 5, pp. 218-221, Sep. 2003.

[71] N. Tas, J. Wissink, L. Sander, T. Lammerink, and M. Elwenspoek, "Modeling, design and testing of the electrostatic shuffle motor," Sensors Actuators A-Physical, vol. 70, pp. 171-178, Oct. 1998. 
[72] H. Rothuizen, U. Drechsler, G. Genolet, W. Haeberle, M. Lutwyche, R. Stutz, R. Widmer, and P. Vettiger, "Fabrication of a micromachined magnetic $x / y / z$ scanning for parallel scanning probe applications," in Proc. 25th Int. Conf. Microelectron. Eng., 2000, pp. 509-512.

[73] J.-J. Choi et al., "Electromagnetic micro x-y stage for probe-based data storage," J. Semicond. Technol. Sci., vol. 1, no. 1, pp. 84-93, Mar. 2001.

[74] M. Despont, U. Drechsler, W. Häberle, M. A. Lantz, and H. E. Rothuizen, "A vibration resistant nanopositioner for mobile parallel-probe storage applications," J. Microelectromechan. Syst., vol. 16, no. 1, pp. 130-139, Feb. 2007.

[75] A. Pantazi, M. A. Lantz, G. Cherubini, H. Pozidis, and E. Eleftheriou, "A servomechanism for a micro-electro-mechanical-system-based scanning-probe data storage device," Nanotechnol., vol. 15, pp. 612-621, Aug. 2004.

[76] Y. B. Gianchandani and K. Najafi, "A silicon micromachined scanning thermal profiler with integrated elements for sensing and actuation," IEEE Trans. Electron Devices, vol. 44, no. 11, pp. 1857-1868, Nov. 1997.

[77] A. Mohamed, H. Elsimary, and M. Ismail, "Analysis, and optimization of a CMOS vertical thermal actuator," in Proc. Symp. Design, Test, Integr. Pack. MEMS/MOEMS, 2003, pp. 214-217.

[78] D. Yan, A. Khajepour, and R. Mansour, "Design and modeling of a MEMS bidirectional vertical thermal actuator," J. Micromech. Microeng., vol. 14, no. 7, pp. 841-850, Jul. 2004.

[79] L.-S. Zheng and M. S.-C. Lu, "A large-displacement CMOS-micromachined thermal actuator with capacitive position sensing," in Proc. 1st IEEE Asian Solid-State Circuits Conf. (A-SSCC), 2005, pp. 89-92.

[80] E. O. Doebelin, Measurement Systems Application and Design, 5th ed. New York: McGraw-Hill, 2004.

[81] R. Brinkerhoff and S. Devasia, "Output tracking for actuator deficient/ redundant systems: Multiple piezoactuator example," AIAA J. Guid., Control, Dyn., vol. 23, no. 2, pp. 370-373, Mar./Apr. 2000.

[82] R. Pedrak, T. Ivanov, K. Ivanova, T. Gotszalk, N. Abedinov, I. W. Rangelowa, K. Edinger, E. Tomerov, T. Schenkel, and P. Hudek, "Micromachined atomic force microscopy sensor with integrated piezoresistive, sensor and thermal bimorph actuator for high-speed tappingmode atomic force microscopy phase-imaging in higher eigenmodes," J. Vacuum Sci. Technol. B, vol. 21, no. 6, pp. 3102-3107, Nov./Dec. 2003.

[83] C. H. Chien, Y. D. Wu, Y. T. Chiou, C. C. Hsieh, Y. C. Chen, T. P. Chen, M. L. Tsai, and C. T. Wang, "Nanoscale deformation measurement by using the hybrid method of gray-level and holographic interferometry," Opt. Lasers Eng., vol. 44, no. 1, pp. 80-91, Jan. 2006.

[84] J. Schneir, T. H. McWaid, J. Alexander, and B. P. Wilfley, "Design of an atomic-force microscope with interferometric position control," $J$. Vacuum Sci. Technol. B, vol. 12, no. 6, pp. 3561-3566, Nov./Dec. 1994.

[85] M. Lantz, G. Binnig, M. Despont, and U. Drechsler, "A micromechanical thermal displacement sensor with nanometer resolution," Nanotechnol., vol. 16, pp. 1089-1094, May 2005.

[86] D. W. Pohl, "Dynamic piezoelectric translation devices," Rev. Sci. Instrum., vol. 58, no. 1, pp. 54-57, Jan. 1987.

[87] Q. Zou, C. Vander Giessen, J. Garbini, and S. Devasia, "Precision tracking of driving waveforms for inertial reaction devices," Rev. Scientific Instrum., vol. 76, no. 2, pp. 1-9, Feb. 2005, Article 023701.

[88] E. Fukada, "History and recent progress in piezoelectric polymers," IEEE Trans. Ultrason., Ferroelectr. Freq. Control, vol. 47, no. 6, pp. 1277-1290, Nov. 2000.

[89] T. Ikeda, Fundamentals of Piezoelectricity. Oxford, U.K.: Oxford Univ. Press, 1996.

[90] IEEE Standard on Piezoelectricity, ANSI/IEEE Std. 976-987, 1988.

[91] I. J. Busch-Vishniac, Electromechanical Sensors and Actuators. Berlin, Germany: Springer-Verlag, 1999.

[92] S. O. R. Moheimani and A. J. Fleming, Piezoelectric Transducers for Vibration Damping and Control. Berlin, Germany: Springer, 2006.

[93] G. Binnig and D. P. E. Smith, "Single-tube three-dimensional scanner for scanning tunneling microscopy," Rev. Sci. Instrum., vol. 57, no. 8, pp. 1688-1689, Aug. 1986.

[94] E. Meyer, H. J. Hug, and R. Bennewitz, Scanning Probe Microscopy. Heidelberg, Germany: Springer, 2004.

[95] W. Gao, R. J. Hocken, J. A. Patten, J. Lovingood, and D. A. Lucca, "Construction and testing of a nanomachining instrument," Prec. Eng., vol. 24, no. 4, pp. 320-328, Oct. 2000.

[96] J. Shim and D. Gweon, "Piezo-driven metrological multiaxis nanopositioner," Rev. Sci. Instrum., vol. 72, no. 11, pp. 4183-4187, 2001.
[97] S. Salapaka and A. Sebastian, "Control of the nanopositioning devices," in Proc. IEEE Conf. Dec. Control, 2003, pp. 3644-3649.

[98] R. G. Carr, "Finite-element analysis of PZT tube scanner motion for scanning tunnelling microscopy," J. Microscopy, vol. 152, pp. 379-385, Nov. 1988.

[99] C. J. Chen, "In situ testing and calibration of piezoelectric tube scanners," Ultramicroscopy, vol. 42-44, pp. 1653-1658, 1992.

[100] C. J. Chen, "Electromechanical deflections of piezoelectric tubes with quartered electrodes," Appl. Phys. Lett., vol. 60, no. 1, pp. 132-134, Jan. 1992.

[101] N. Tamer and M. Dahleh, "Feedback control of piezoelectric tube scanners," in Proc. 33rd IEEE Conf. Dec. Control, 1994, pp. 1826-1831.

[102] M. S. Tsai and J. S. Chen, "Robust tracking control of a piezoactuator using a new approximate hysteresis model," J. Dyn. Syst., Meas., Control, vol. 125, pp. 96-102, Mar. 2003.

[103] M. E. Taylor, "Dynamics of piezoelectric tube scanners for scanning probe microscopy," Rev. Sci. Instrum., vol. 64, no. 1, pp. 154-158, 1993.

[104] T. Ohara and K. Youcef-Toumi, "Dynamics and control of piezotube actuators for subnanometer precision applications," in Proc. Amer. Control Conf., 1995, pp. 3808-3812.

[105] S. Yang and W. Huang, "Transient response of a piezoelectric tube scanner," Rev. Sci. Instrum., vol. 68, no. 12, pp. 4483-4487, 1997.

[106] J. Tapson and J. R. Greene, "The resonant behavior of segmented piezoceramic tubes," Rev. Sci. Instrum., vol. 68, no. 7, pp. 2797-2799, 1997.

[107] S. Yang and W. Huang, "Three-dimensional displacements of a piezoelectric tube scanner," Rev. Sci. Instrum., vol. 69, no. 1, pp. 226-229, 1998.

[108] S. Bonnail, D. Tonneau, G. A. Capolino, and H. Dallaporta, "Dynamic and static responses of a piezoelectric actuator at nanometer scale elongation," in Proc. IEEE Ind. Appl. Conf., 2000, pp. 293-298.

[109] T. Fett and G. Thun, "Determination of room-temperature tensile creep of PZT,” J. Mater. Sci. Lett., vol. 17, no. 22, pp. 1929-1931, 1998.

[110] R. S. Robinson, "Interactive computer correction of piezoelectric creep in scanning tunneling microscopy images," J. Comput.-Assisted Microscopy, vol. 2, no. 1, pp. 53-58, 1996.

[111] H. Jung, J. Y. Shim, and D. Gweon, "New open-loop actuating method of piezoelectric actuators for removing hysteresis and creep," Rev. Sci. Instrum., vol. 71, no. 9, pp. 3436-3440, Sep. 2000.

[112] L. E. Malvern, Ed., Introduction to the Mechanics of a Continuous Medium. Englewood Cliffs, NJ: Prentice-Hall, 1969, ch. 6, pp. 313-319.

[113] R. C. Smith, Z. Ounaies, and R. Wieman, "A model for rate-dependent hysteresis in piezoceramic materials operating at low frequencies," ICASE/NASA, Tech. Rep. NASA/CR-2001-211062 ICASE report 2001-26, 2001.

[114] D. Song and C. J. Li, "Modeling of piezo actuator's nonlinear and frequency dependent dynamics," Mechatron., vol. 9, pp. 391-410, 1999.

[115] H. Hu and R. B. Mrad, "On the classical Preisach model of hysteresis in piezoceramic actuators," Mechatron., vol. 13, pp. 85-94, 2003.

[116] R. B. Mrad and H. Hu, "Dynamic modeling of hysteresis in piezoceramics," in Proc. IEEE/ASME Int. Conf. Adv. Intell. Mechatron., 2001, pp. 510-515.

[117] P. Ge and M. Jouaneh, "Tracking control of a piezoceramic actuator," IEEE Trans. Control Syst. Technol., vol. 4, no. 3, pp. 209-216, May 1996.

[118] J. A. Main and E. Garcia, "Piezoelectric stack actuators and control system design: Strategies and pitfalls," J. Guid., Control Dyn., vol. 20, no. 3, pp. 479-485, May/Jun. 1997.

[119] D. Croft and S. Devasia, "Hysteresis and vibration compensation for piezoactuators," J. Guid., Control, Dyn., vol. 21, no. 5, pp. 710-717, Sep./Oct. 1998.

[120] I. Mayergoyz, Mathematical Models of Hysteresis. Berlin, Germany: Springer-Verlag, 1991.

[121] M. Goldfarb and N. Celanovic, "A lumped parameter electromechanical model for describing the nonlinear behavior of piezoelectric actuators," J. Dyn. Syst., Meas., Control, vol. 119, pp. 478-485, Sep. 1997.

[122] P. Ge and M. Jouaneh, "Modeling hysteresis in piezoceramic actuators," Precision Eng., vol. 17, pp. 211-221, 1995.

[123] R. B. Mrad and H. Hu, "A model for voltage to displacement dynamics in piezoceramic actuators subject to dynamic voltage excitations," IEEE/ASME Trans. Mechatron., vol. 7, no. 4, pp. 479-489, Dec. 2002. 
[124] S. H. Lee and T. J. Royston, "Modeling piezoceramic transducer hysteresis in the structural vibration control problem," J. Acoust. Soc. Amer., vol. 108, no. 6, pp. 2843-2855, Dec. 2000.

[125] S. H. Lee, T. J. Royston, and G. Friedman, "Modeling and compensation of hysteresis in piezoceramic transducers for vibration control," $J$. Intell. Mater. Syst. Structures, vol. 11, pp. 781-790, Oct. 2000.

[126] S. Mittal and C. Menq, "Hysteresis compensation in electromagnetic actuators through Preisach model inversion," IEEE/ASME Trans. Mechatron., vol. 5, no. 4, pp. 394-409, Dec. 2000.

[127] R. B. Gorbet, "Control of hysteresis systems with preisach representations," Ph.D. dissertation, Dept. Electr. Comput. Eng., Univ. Waterloo, Waterloo, ON, Canada, 1997.

[128] P. Nakmahachalasint, K. D. T. Ngo, and L. Vu-Quoc, "A static hysteresis model for power ferrites," IEEE Trans. Power Electron., vol. 17, no. 4, pp. 453-460, Jul. 2002.

[129] R. Comstock, "Charge control of piezoelectric actuators to reduce hysteresis effect," U.S. Patent 4263 527, Apr. 21, 1981.

[130] C. Newcomb and I. Flinn, "Improving the linearity of piezoelectric actuators using charge feedback," Electron. Lett., vol. 18, no. 11, pp. 442-444, May 1982.

[131] J. A. Main and E. Garcia, "Design impact of piezoelectric actuator nonlinearities," AIAA J. Guid. Control Dyn., vol. 20, no. 2, pp. 327-332, Mar./Apr. 1997.

[132] J. A. Main, E. Garcia, and D. V. Newton, "Precision position control of piezoelectric actuators using charge feedback," AIAA J. Guid., Control Dyn., vol. 18, no. 5, pp. 1068-1073, 1995.

[133] A. J. Fleming and S. O. R. Moheimani, "Improved current and charge amplifiers for driving piezoelectric loads, and issues in signal processing design for synthesis of shunt damping circuits," J. Intell. Mater. Syst. Structures, vol. 15, no. 2, pp. 77-92, Feb. 2004.

[134] A. J. Fleming and S. O. R. Moheimani, "A grounded load charge amplifier for reducing hysteresis in piezoelectric tube scanners," Rev. Sci. Instrum., vol. 76, no. 7, pp. 1-5, 2005.

[135] A. J. Fleming and S. O. R. Moheimani, "Sensorless vibration suppression and scan compensation for piezoelectric tube nanopositioners," IEEE Trans. Control Syst. Technol., vol. 14, no. 1, pp. 33-44, Jan. 2006.

[136] B. Bhikkaji, M. Ratnam, A. J. Fleming, and S. O. R. Moheimani, "High-performance control of a PZT scanner," presented at the 4th IFAC Symp. Mechatron. Syst., Heidelberg, Germany, 2006.

[137] D. Niederberger, "Smart Damping Materials using Shunt Control," Ph.D. dissertation, Dept. Electr. Eng., Swiss Federal Inst. Technol. (ETH), Zurich, Switzerland, 2005.

[138] M. J. Balas, "Active control of flexible systems," J. Opt. Theory Appl., vol. 25, no. 3, pp. 416-436, Jul. 1978.

[139] O. M. El Rifai and K. Youcef-Tomi, "Coupling in piezoelectric tube scanners used in scanning probe microscopes," in Proc. Amer. Control Conf., 2001, pp. 3251-3255.

[140] S. Tien, Q. Zou, and S. Devasia, "Iterative control of dynamics-coupling-caused errors in piezoscanners during high-speed AFM operation," IEEE Trans. Control Syst. Technol., vol. 13, no. 6, pp. 921-931, Nov. 2005.

[141] E. Kouno, "A fast response piezoelectric actuator for servo correction of systematic errors in precision machining," Ann. CIRP, vol. 33, no. 1, pp. 369-372, 1982.

[142] J. F. Cuttino, A. C. Miller, and D. E. Schinstock, "Performance optimization of a fast tool servo for single-point diamond turning machines," IEEE/ASME Trans. Mechatron., vol. 4, no. 2, pp. 169-179, Jun. 1991.

[143] E. Kouno, "PID control," IEEE Control Syst. Mag., vol. 26, no. 1, pp. 30-31, Jan. 2006

[144] R. C. Barrett and C. F. Quate, "Optical scan-correction system applied to atomic force microscopy," Rev. Sci. Instrum., vol. 62, no. 6, pp. 1393-1399, Jun. 1991.

[145] K. K. Tan, T. H. Lee, and H. X. Zhou, "Micro-positioning of linearpiezoelectric motors based on a learning nonlinear PID controller," IEEE/ASME Trans. Mechatron., vol. 6, no. 4, pp. 428-36, Dec. 2001.

[146] Y. Okazaki, "A micro-positioning tool post using a piezoelectric actuator for diamond turning machines," Precision Eng., vol. 12, pp. 151-156, Jul. 1990.

[147] H. Numasato and M. Tomizuka, "Settling control and performance of dual-actuator system for hard disk drives," in Proc. Amer. Control Conf., 2001, pp. 2779-2785.

[148] K. Leang and S. Devasia, "Hysteresis, creep, and vibration compensation for piezoactuators: Feedback and feedforward control," in Proc. 2nd IFAC Conf. Mechatron. Syst., 2002, pp. 283-289.

[149] C. J. Li, H. S. M. Beigi, S. Li, and J. Liang, "Nonlinear piezo-actuator control by learning self tuning regulator," J. Dyn. Syst., Meas. Control, vol. 115 , pp. $720-723$, Dec. 1993.
[150] X. Tan and J. S. Baras, "Adaptive identification and control of hysteresis in smart materials," IEEE Trans. Autom. Control, vol. 50, no. 6, pp. 827-839, Jun. 2005.

[151] J. M. Cruz-Hernandez and V. Hayward, "Phase control approach to hysteresis reduction," IEEE Trans. Control Syst. Technol., vol. 9, no. 1, pp. 17-26, Jan. 2001.

[152] D. Croft, D. McAllister, and S. Devasia, "High-speed scanning of piezo-probes for nano-fabrication," ASME J. Manuf. Sci. Eng., vol. 120, no. 3, pp. 617-622, Aug. 1998.

[153] S. Bashash and N. Jalili, "Intelligence rules of hysteresis in the feedforward trajectory control of piezoelectrically-driven nanostagers," $J$. Micromechan. Microeng., vol. 17, no. 2, pp. 342-349, Feb. 2007.

[154] H. Hu, H. M. S. Georgiou, and R. Ben-Mrad, "Enhancement of tracking ability in piezoceramic actuators subject to dynamic excitation conditions," IEEE/ASME Trans. Mechatron., vol. 10, no. 2, pp. 230-9, Apr. 2005.

[155] K. Kuhnene and H. Janocha, "Inverse feedforward controller for complex hysteretic nonlinearities in smart-material systems," Control Intell. Syst., vol. 29, no. 3, pp. 74-83, 2001.

[156] H. Janocha and K. Kuhnen, "Real-time compensation of hysteresis and creep in piezoelectric actuators," Sensors Actuators A, vol. 79, pp. 83-89, 2000.

[157] H. J. M. T. A. Adriaens, W. L. de Koning, and R. Banning, "Modeling piezoelectric actuators," IEEE/ASME Trans. Mechatron., vol. 5, no. 4, pp. 331-41, Dec. 2000

[158] S. B. Jung and S. W. Kim, "Improvement of scanning accuracy of PZT piezoelectric actuators by feed-forward model-reference control," Precision Eng., vol. 17, pp. 49-55, 1994.

[159] J. M. Cruz-Hernandez and V. Hayward, "An approach to reduction of hysteresis in smart materials," in Proc. IEEE Int. Conf. Robot. Automat., 1998, pp. 1510-1515.

[160] D. Croft, S. Stilson, and S. Devasia, "Optimal tracking of piezo-based nano-positioners," J. Nanotechnol., vol. 10, pp. 201-208, Jun. 1999.

[161] J. S. Dewey, K. Leang, and S. Devasia, "Experimental and theoretical results in output- trajectory redesign for flexible structures," ASME J. Dyn. Syst., Meas. Control, vol. 120, no. 4, pp. 456-461, 1998.

[162] S. Devasia, "Should model-based inverse inputs be used as feedforward under plant uncertainty?," IEEE Trans. Autom. Control, vol. 47, no. 11, pp. 1865-1871, Nov. 2002.

[163] G. Schitter and A. Stemmer, "Model-based signal conditioning for high-speed atomic force and friction force microscopy," Microelectron. Eng., vol. 67-68, pp. 938-944, 2003.

[164] G. Schitter and A. Stemmer, "Identification and open-loop tracking control of a piezoelectric tube scanner for high-speed scanning-probe microscopy," IEEE Trans. Control Syst. Technol., vol. 12, no. 3, pp. 449-54, May 2004

[165] Q. Zou and S. Devasia, "Preview-based optimal inversion for output tracking: Application to scanning tunneling microscopy," IEEE Trans. Control Syst. Technol., vol. 12, no. 3, pp. 375-386, May 2004.

[166] Y. Li and J. Bechhoefer, "Feedforward control of a closed-loop piezoelectric translation stage for atomic force microscope," Rev. Sci. Instrum., vol. 78, pp. 1-8, Jan. 2007

[167] Y.Zhao and S. Jayasuriya, "Feedforward controllers and tracking accuracy in the presence of plant uncertainties," ASME J. Dyn. Syst., Meas. Control, vol. 117, pp. 490-495, Dec. 1995.

[168] G. M. Clayton, S. Tien, A. J. Fleming, S. O. R. Moheimani, and S Devasia, "Hysteresis and vibration compensation in piezoelectric actuators by integrating charge control and inverse feedforward," presented at the 4th IFAC Symp. Mechatron. Syst., Heidelberg, Germany, 2006.

[169] T. Tsao and M. Tomizuka, "Adaptive zero phase error tracking algorithm for digital control," ASME J. Dyn. Syst., Meas. Control, vol. 109, no. 2, pp. 349-354, Dec. 1987.

[170] J. Ghosh and B. Paden, "A pseudo-inverse based iterative learning control," IEEE Trans. Autom. Control, vol. 47, no. 5, pp. 831-837, May 2002.

[171] J. D. Rasmussen, T.-C. Tsao, R. D. Hanson, and S. G. Kapoor, "A piezoelectric tool servo system for variable depth of cut machining," ASME Precision Machin.: Technol. Mach. Developm. Improvement, vol. 58, pp. 119-130, 1992.

[172] G. Tao and P. V. Kokotovic, "Adaptive control of plants with unknown hysteresis," IEEE Trans. Autom. Control, vol. 40, no. 1, pp. 200-212, Feb. 1995.

[173] K. K. Leang and S. Devasia, "Design of hysteresis-compensating iterative learning control: Application to atomic force microscopes," Mechatron., vol. 16, no. 3-4, pp. 141-158, Apr./May 2006.

[174] M. Hu, H. Du, and S.-F. Ling, "Motion control of an electrostrictive actuator," in Proc. SPIE Smart Structures Devices, 2001, pp. 321--327. 
[175] R. V. Iyer, X. Tan, and P. S. Krishnaprasad, “Approximate inversion of the Preisach hysteresis operator with application to control of smart actuators," IEEE Trans. Autom. Control, vol. 50, no. 6, pp. 798-810, Jun. 2005

[176] G. Schitter, R. W. Stark, and A. Stemmer, "Fast contact-mode atomic force microscopy on biological specimen by model-based control," $U l$ tramicroscopy, vol. 100, no. 3-4, pp. 253-257, Aug. 2004.

[177] G. D. Martin, "On the control of flexible mechanical systems," Ph.D. dissertation, Dept. Aeronautics Astronautics, Stanford Univ., Stanford, CA, 1978.

[178] C. J. Goh and T. K. Caughey, "On the stability problem caused by finite actuator dynamics in the collocated control of large space structures," Int. J. Control, vol. 41, no. 3, pp. 787-802, 1985.

[179] J. L. Fanson and T. K. Caughey, "Positive position feedback-control for large space structures," AIAA J., vol. 28, no. 4, pp. 717-724, Apr. 1990.

[180] J. J. Dosch, D. J. Inman, and E. Garcia, "A self-sensing piezoelectric actuator for collocated control," J. Intell. Mater. Structures, vol. 3, no. 1, pp. 166-185, 1992.

[181] S. O. R. Moheimani, B. J. G. Vautier, and B. Bhikkaji, "Multivariable PPF control of an active structure," in Proc. IEEE CDC-ECC, 2005, pp. 6824-6829.

[182] M. Ratnam, B. Bhikkaji, A. J. Fleming, and S. O. R. Moheimani, "PPF control of a piezoelectric tube scanner," in Proc. IEEE CDC-ECC, 2005, pp. 1168-1173.

[183] B. Bhikkaji, M. Ratnam, and S. O. R. Moheimani, "PVPF control of piezoelectric tube scanners," Sensors Actuators A-Phys., vol. 132, no. 2, pp. 700-712, Apr. 2007.

[184] R. L. Forward, "Electronic damping of vibrations in optical structures," J. Appl. Opt., vol. 18, no. 5, pp. 690-697, 1979.

[185] N. W. Hagood and A. von Flotow, "Damping of structural vibrations with piezoelectric materials and passive electrical networks," J. Sound Vibration, vol. 146, no. 2, pp. 243-268, 1991.

[186] G. A. Lesieutre, "Vibration damping and control using shunted piezoelectric materials," Shock Vibration Dig., vol. 30, pp. 187-195, 1998.

[187] S. O. R. Moheimani, "A survey of recent innovations in vibration damping and control using shunted piezoelectric transducers," IEEE Trans. Control Syst. Technol., vol. 11, no. 4, pp. 482-494, Jul. 2003.

[188] A. J. Fleming, S. Behrens, and S. O. R. Moheimani, "Synthetic impedance for implementation of piezoelectric shunt damping circuits," Electron. Lett., vol. 36, no. 18, pp. 1525-1526, 2000.

[189] S. O. R. Moheimani, A. J. Fleming, and S. Behrens, "On the feedback structure of wideband piezoelectric shunt damping systems," Smart Mater. Structures, vol. 12, no. 1, pp. 49-56, Feb. 2003.

[190] G. Clayton and S. Devasia, "Image-based control of dynamic effects in scanning tunneling microscopes," Nanotechnol., vol. 16, no. 6, pp. 809-818, Jun. 2005.

[191] R. V. Lapshin, "Automatic lateral calibration of tunneling microscope scanners," Rev. Sci. Instrum., vol. 69, no. 9, pp. 3268-3276, Sep. 1998.

[192] R. V. Lapshin, "Automatic drift elimination in probe microscope images based on techniques of counter-scanning and topography feature recognition," Meas. Sci. Technol., vol. 18, no. 3, pp. 907-927, Mar. 2007.

[193] S. Hutchinson, G. Hager, and P. Corke, "A tutorial on visual servo control,” IEEE Trans. Robot. Autom., vol. 12, pp. 651-670, Oct. 1996.

[194] T. Sulchek, R. Hsieh, J. D. Adams, S. C. Minne, C. F. Quate, and D. M. Adderton, "High-speed atomic force microscopy in liquid," Rev. Sci. Instrum., vol. 71, no. 5, pp. 2097-2099, May 2000.

[195] K. Wilder, H. T. Soh, A. Atalar, and C. F. Quate, "Nanometer-scale patterning and individual current controlled lithography using multiple scanning probes," Rev. Sci. Instrum., vol. 70, pp. 2822-2827, 1999.

[196] B. A. Wallman and P. G. Crawley, "Nanolithography requirements-An equipment manufacturers view," in Proc. NATO Adv. Workshop Nanolithography: A Borderland Between STM, EB, IB, X-Ray Lithographies, 1993, pp. 95-101.

[197] N. Shimizu, T. Kimura, T. Nakamura, and I. Umebu, "An ultrahigh vacuum scanning tunneling microscope with a new inchworm mechanism," J. Vacuum Sci. Technol. A, vol. 8, no. 1, pp. 333-335, 1990.

[198] D. Jeon and R. F. Willis, "Inchworm controller for final approach in a scanning tunneling microscope," J. Vacuum Sci. Technol. A, vol. 9, no. 4, pp. 2418-2419, Aug. 1991.

[199] D. M. Eigler and E. K. Schweizer, "Positioning single atoms with a scanning tunneling microscope," Nature, vol. 344, pp. 524-526, 1990.

[200] S. C. Minne, G. Yaraliuglu, S. R. Manalis, J. D. Adams, J. Zesch, A Atalar, and C. F. Quate, "Automated parallel high-speed atomic force microscopy," Appl. Phys. Lett., vol. 72, no. 18, pp. 2240-2342, 1998.

[201] A. L. D. Lozanne, W. F. Smith, and E. E. Ehrichs, "Direct writing with a combined stm/sem system," in Proc. NATO Adv. Workshop Nanolithography: Borderland Between STM, EB, IB, X-Ray Lithographies, 1993, pp. 159-174.
[202] J. Aizenberg, A. J. Black, and G. M. Whitesides, "Control of crystal nucleation by patterned self-assembled monolayers," Nature, vol. 398, pp. 495-498, 1999.

[203] D. C. Coffey and D. S. Ginger, "Patterning phase separation in polymer films with dip-pen nanolithography," J. Amer. Chem. Soc., vol. 127, no. 13, pp. 4564-4565, 2005.

[204] S. W. Chung, D. S. Ginger, M. Morales, Z. Zhang, V. Chandrasekhar, M. A. Ratner, and C. A. Mirkin, "Top-down meets bottom-up: Dip-pen nanolithography and DNA-directed assembly of nanoscale electrical circuits," Small, vol. 1, no. 1, pp. 64-69, 2005.

[205] C. Park, J. Yoon, and E. L. Thomas, "Enabling nanotechnology with self assembled block copolymer patterns," Polymer, vol. 44, no. 22, pp. 6725-6760, Oct. 2003

[206] A. Piazzi and A. Visioli, "Minimum-time system-inversion-based motion planning for residual vibration reduction," IEEE/ASME Trans. Mechatron., vol. 5, no. 1, pp. 12-22, Mar. 2000.

[207] H. Perez and S. Devasia, "Optimal output-transitions for linear systems," Automatica, vol. 39, no. 2, pp. 181-192, Feb. 2003.

[208] H. Perez, Q. Zou, and S. Devasia, "Design and control of optimal scantrajectories: Scanning tunneling microscope example," ASME J. Dyn. Syst. Meas. Control, vol. 126, no. 1, pp. 187-197, Mar. 2004.

[209] P. Mortensen, "Will AFM data-storage replace optical methods," Laser Focus World, vol. 30, no. 11, p. 16, Nov. 1994.

[210] P. Vettiger, G. Cross, M. Despont, U. Drechsler, U. Dürig, B. Gotsmann, W. Häberle, M. A. Lantz, H. E. Rothuizen, R. Stutz, and G. K. Binnig, "The 'millipede'-nanotechnology entering data storage," IEEE Trans. Nanotechnol., vol. 1, no. 1, pp. 39-55, Mar. 2002.

[211] E. Eleftheriou, T. Antonakopoulos, G. K. Binnig, G. Cherubini, M. Despont, A. Dholakia, U. Dürig, M. A. Lantz, H. Pozidis, H. E. Rothuizen, and P. Vettiger, "Millipede-A MEMS-based scanning-probe data-storage system," IEEE Trans. Magn., vol. 39, no. 2, pt. 1, pp. 938-945, Mar. 2003.

[212] E. Eleftheriou, P. Baechtold, G. Cherubini, A. Dholakia, C. Hagleitner, T. Loeliger, A. Pantazi, H. Pozidis, T. Albrecht, G. Binnig, M. Despont, U. Drechsler, U. T. Duerig, B. Gotsmann, D. Jubin, W. Haeberle, M. A. Lantz, H. E. Rothuizen, R. Stutz, P. Vettiger, and D. W. Wiesmann, "A nanotechnology-based approach to data storage," in Proc. 29th Int. Conf. Very Large Data Bases (VLDB), 2003, pp. 3-7.

[213] H. Rothuizen, M. Despont, U. Drechsler, G. Genolet, W. Häberle, M. Lutwyche, R. Stutz, and P. Vettiger, "Compact copper/epoxy-based micromachined electromagnetic scanner for scanning probe applications," in Proc. 15th IEEE Int. Conf. Micro Electro Mechan. Syst., 2002, pp. 582-585.

[214] M. Lutwyche et al., "Planar micromagnetic x/y/z scanner with five degrees of freedom," in Magnetic Materials, Processes and Devices: Applications to Storage and Micromechanical Systems (MEMS), L. Romantkiv, S. Krongeld, and C. H. Ahn, Eds. Pennington, NJ: Electrochemical Society, 1999, vol. 98-20, pp. 423-433.

[215] E. Sarajlic et al., "Versatile trench isolation technology for the fabrication of microactuators," Microelectron. Eng., vol. 67, pp. 430-437, 2003.

[216] F. Laermer et al., "Bosch deep silicon etching: Improving uniformity and etch rate for advanced MEMS applications," in Proc. IEEE 12th Int. Conf. Micro Electro Mechan. Syst., 1999, pp. 211-216.

[217] G. Gibson, T. I. Kamins, M. S. Keshner, S. L. Neberhuis, C. M. Perlov, and C. C. Yang, "Ultra-high density storage device," U.S. Patent 5557 596, Sep. 17, 1996.

[218] H. Pozidis, W. Haeberle, D. W. Wiesmann, U. Drechsler, M. Despont, T. Albrecht, and E. Eleftheriou, "Demonstration of thermomechanical recording at $641 \mathrm{gbit} / \mathrm{in}^{2}$," IEEE Trans. Magn., vol. 40, no. 4, pp. 2531-2536, Jul. 2004.

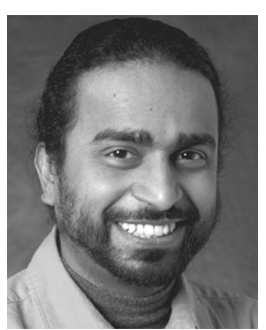

Santosh Devasia (SM'03) received the B.Tech. (hon.) degree from the Indian Institute of Technology, Kharagpur, India, in 1988, and the M.S. and $\mathrm{Ph} . \mathrm{D}$. degrees in mechanical engineering from the University of California, Santa Barbara, in 1990 and 1993, respectively.

He is a Professor with the Mechanical Engineering Department, the University of Washington, Seattle, where he has been since 2000. From 1994 to 2000, he taught in the Mechanical Engineering Department, the University of Utah, Salt Lake City. His current research interests include inversion-based control theory and applications such as high-precision positioning systems for atomic force microscopes and scanning tunneling microscopes used in nanotechnology, biomedical applications such as the imaging of human cells to investigate cell locomotion, and control of distributed systems such as air traffic management. 
Dr. Devasia is an Associate Editor for the ASME Journal of Dynamic Systems, Measurement, and Control and the IEEE TRANSACTIONS ON CONTROL SYSTEMS TECHNOLOGY.

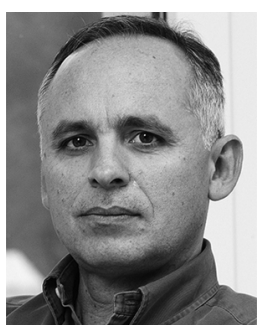

Evangelos Eleftheriou (F’02) received the B.S. degree in electrical engineering from the University of Patras, Patras, Greece, in 1979, and the M.Eng. and $\mathrm{Ph} . \mathrm{D}$. degrees in electrical engineering from Carleton University, Ottawa, ON, Canada, in 1981 and 1985, respectively.

In 1986, he joined the IBM Zurich Research Laboratory, Zurich, Switzerland, where he worked on various projects related to wired and wireless communications, magnetic recording, and probe storage. He currently manages the laboratory's Advanced Storage Technologies Group.

Dr. Eleftheriou was co-recipient of the Eduard Rhein Technology Award in 2005 and a co-recipient of the 2003 IEEE Communications Society Leonard G. Abraham Prize Paper Award. In 2005, he became an IBM Fellow and was elected to the IBM Academy of Technology.

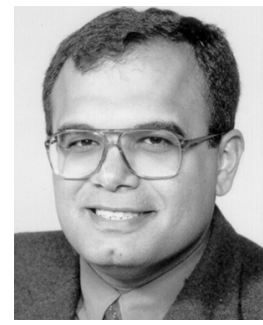

S. O. Reza Moheimani (SM'00) received the Ph.D. degree from University of New South Wales, Australian Defence Force Academy, Canberra, Australia, in 1996.

Following a research position at the same institution, he joined the University of Newcastle, Callaghan, Australia, in 1997, where he is currently an Associate Professor with the School of Electrical Engineering and Computer Science, an Assistant Dean of Research (Engineering), the head of the Laboratory for Dynamics and Control of NanoSystems, and an Associate Director of the ARC Centre of Excellence for Complex Dynamic Systems and Control, an Australian Government Centre of Excellence. He is an Associate Editor of several international journals including the IEEE TRANSACTIONS ON CONTROL SySTEMS TECHNOLOGY and has chaired a number of international workshops and conferences. He has published two books, several edited volumes, and over 150 refereed articles in the areas of robust control and estimation, smart structures, active noise and vibration control, mechatronic systems, and nanotechnology. 